\title{
Über die Wirkung des Novokains anf den normalen und den tetanusstarren Skelettmuskel und über die Entstehung der lokalen Muskelstarre beim Wund- starrkrampf.
}

\author{
G. Liljestrand (Stockholm) und R. Magnus.
}

(Aus dem pharmakologischen Institut der Reichsuniversität Utrecht.)

Mit 1 Textabbildung.

(Eingegangen am 5. April 1919.)

Inhalt. Seite

I. Einleitung. . . . . . . . . . . . . . . . . 168

II. Die Wirkung des Novokains auf den Skelettmuskel im Zustand

der Enthirnungsstarre. . . . . . . . . . . . . 171

III. Die Wirkung kleiner Novokaindosen auf den Skelettmuskel nor-

maler Katzen . . . . . . . . . . . . . . . . . . . . . . . 183

IV. Die Wirkung des Novokains auf den tetanusstarren Skelettmuskel 187

V. Weitere Beobachtungen über die tetanische Muskelstarre . . . . 202

VI. Zusammenfassung der Ergebnisse . . . . . . . . . . 206

\section{Einleitung.}

Im Jahre 1916 haben E. Meyer und L. Weiler ${ }^{1}$ ) einen Fall von Starre der Bauchmuskulatur beschrieben, die sich im Anschluss an eine Tetanusinfektion entwickelte und noch nach 2 Jahren fortbestand. Diese Statre blieb nach intralumbaler Einspritzung von $0,02 \mathrm{~g}$ Stovain in den Lumbalsack; wonach eine Hautanästhesie bis zur Mámmillarlinie (entsprechend dem fünften Thorakalsegment) auftrat, unverändert bestehen, während die Nerven für die Bauchmuskeln nicht höher als aus dem siebenten Thorakalsegment entspringen. Ebensowenig liess sie sich durch Einspritzung von $1 \mathrm{ccm}$ Curarelösung (entsprechend 8.5 mg Curare bzw. $2 \mathrm{mg}$ Curarin) lösen. Dagegen schwand die Starre nach Einspritzung von $10-15 \mathrm{ccm}$ einer $1 \%$ igen Norokainlösung in den Muskel, wonach aber die direkte elektrische Muskelerregbarkeit unverändert blieb. Die mit dem ballistischen Elastometer gemessene Muskelhärte nahm dabei deutlich ab. Während die Haut nicht hyperästhetisch war, empfand der Patient den Einstich der Nadel in den Muskel als äusserst schmerzhaft. In zwei weiteren

1) Erich Meyer und Leo Weiler, Über Muskelstarre und Koordinationsstörung bei Tetanus. Münchner med. Wochenschr. $1916 \mathrm{~S} .1525$. 
Fällen von frischem Tetanus komnten Meyer und Weiler durch Einspritzen von 10-15 ccm 1\% igem Novokain in die Masseteren ein Nachlassen der Kieferklemme erzielen. Sie schliessen aus diesen Beobachtungen, dass die tetanische Muskelverkürzung nicht auf myositischen Schwielen beruhen kann, dass es sich nicht um eine vom zentralen Nervensystem hervorgerufene Kontraktion handelt, und dass die Starre nicht durch aktive Muskelkontraktion hervorgerufen wird, vielmehr müsse es sich um einen eigenartigen, von der gewöhnlichen Kontraktion verschiedenen Verkürzungszustand bandeln. Novokain (und Kokain) sollen die Kontraktilität des Muskels unverändert lassen, aber. die Elastizität desselben in besonderer Weise verändern.

A. Fröhlich und H. H. Meyer ${ }^{1}$ ) haben in einer Kritik dieser Arbeit darrauf bingewiesen, dass es durch Injektion von Curare in den Warmblütermuskei nicht gelingt, eine Lokalcuraresierung herbeizuführen, solange es nicht zur Ailgemeinvergiftung kommt. Ferner zeigten sie, wie schon früher Finkelstein ${ }^{2}$ ) and Baglioni und Pilotti ${ }^{3}$ ), dass bei der Lumbalanästhesie durch Stovain die verschiedenen Gefühlsqualitäten nicht gleichmässig aufgehoben werden, dass daher bei vorhandener Analgesie die übrigen sensiblen Bahnen und vor allem die motorischen Wurzeln nicht gelähmt zu sein brauchen. Daher sei in den Versuchen von E. Meyer und Weiler weder durch Curare noch durch Lumbalinjektion von Stovain die zentrale Innervation des Muskels ausgeschaltet. Ferner haben Fröhlich und Meyer drei Katzen mit Tetanustoxin vergiftet und nach eingetretener lokaler Muskelstarre Novokain injiziert. Bei einer Katze von $650 \mathrm{~g}$ schwand nach Einspritzung von $4-4 \frac{1}{2} \mathrm{ccm} 1 \%$ igem Novokain die Tetanusstarre des Gastrocnemius teilweise, aber nicht gänzlich. Bei einer Katze von $3000 \mathrm{~g}$ wurde durch $4 \mathrm{ccm} 1 \%$ igem Novokain die Starre des Gastrocnemius nicht vermindert. Zwei Tiere wurden nach Eintritt der lokalen Muskelstarre dezerebriert, so dass die tetanischen Muskeln unter den kombinierten Einfluss der tetanischen und der Enthirnungsstarre gerieten. Einspritzung von $1 \%$ Novokain in die tetanusstarren Gastrocnemii hob die Starre nicht auf, während diejenigen (Oberschenkel-)Muskeln, welche sich ńur unter dem Einfluss der Enthirnungs-

1) A. Fröhlich und H. H. Meyer, Über die Muskelstarre bei der Tetanusvergiftung. Münchner med. Wochensohr. 1917 S. 289.

2) R. Finkelstein, Neurologische Beobachtungen und Untersuchungen bei der Rückenmarksanästhesie mittels Kokain und Stovain. Münchner med. Wochenschr. 1906 S. 397.

3) S. Baglioni und G. Pilotti, Neurologische;Untersuchungen bei der menschlichen Lumbalanästhesie mittels Stovain. Zentraibl. f. Physiol. Bd. 23 S. 869.1909. 
starre befanden, durch die gleiche Lösung zur Erschlaffung gebracht wurden. Meyer und Fröhlich schliessen hieraus, dass durch die von E. Meyer und Weiler verwendeten Novokaindosen die gesamte sensible und motorische Innervation des Muskels bei erhaltener Muskelerregbarkeit ausgeschaltet worden sei. Die tetanische Muskelstarre wird durch eine Einwirkung des Tetanusgiftes aùf das Rückenmark verursacht; nach einigen Tagen kann sich hieran eine durch die Dauerverkürzung bedingte, mit Atrophie einhergehende sekundäre myjogene Verkürzung anschliessen. In dem Falle von E. Meyer und Weiler habe es sich nur um die erste Art der Verkürzung gehandelt, die also naeh Lähmung aller Nerven durch Novokain aufhören musste.

In einer weiteren Mitteilung ${ }^{1}$ ) haben dann Erich Meyer und Weiler zu dieser Kritik Stellung genommen. Sie teilen mit, dass man beim Menschen durch intramuskuläre Curareinjektion eine Parese hervorrufen kann, und geben an, dass nach der von ihnen verwendeten Stovaindosis $(0,04$ statt der in der ersten Arbeit genannten $0,02 \mathrm{~g})$ völlige Muskellähmung im anästhetischen Bezirk nach Lumbalinjektion eingetreten sei. Ferner berichten sie über einen weiteren Tetanusfall, an welchem genauere Beobachtungen gemacht werden konnten. Es handelte sich um eine Starre des linken Biceps. Die indirekte faradische Erregbarkeit wurde durch Reizung des Plexus brachialis bestimmt. Nach Einspritzung von $20 \mathrm{ccm} 1 \%$ igem Novokain in den linken Biceps erfolgte vollständige Lösung der Starre, dabei blieb aber die aktive Beweglichkeit und die grobe Kraft unvermindert erhalten. Ebensowenig wurde die indirekte faradjsche Erregbarkeit herabgesetzt. Auch auf der gesunden rechten Seite erfolgte auf Einspritzung derselben Novokaindose in den Biceps keine Abnabme der indirekten Erregbarkeit. In den starren Muskel konnten sogar bis zu $60 \mathrm{ccm}$ der Novokainlösung injiziert werden, ohne dass sich grobe Kraft und indirekte Erregbarkeit änderten. Erst auf $80 \mathrm{ccm}$ erfolgte Abnahme der indirekten Erregbarkeit und der groben Kraft. Der durch Einspritzung von $50 \mathrm{ccm}$ Novokainlösung zur Erschlaffung gebrachte tetanische Biceps beteiligte sich danach noch an einem allgemeinen tetanischen Anfall. Durch Novokaineinspritzung in die Masseteren liess sich die Kieferklemme lösen, dabei konnte der Patient aber noch kräftige Kaubewegungen ausführen. Nach der Ansicht von Meyer und Weiler ist also Novokain ohne Wirkung auf die Kontraktilität des Muskels. Wo das Mittel angreift, lassen sie unentschieden, weisen-aber auf die Möglichkeit hin, dass es auf die von Boeke im

1) Erich Meyer und L. Weiler, Weitere Untersuchungen über die tetanische Muskelverkürzung. Münchner med. Wochenschr. 1917 S. 1569. 
Muskel beschriebenen akzessorischen Nervenendigungen einwirken könne. Sicher ist aber nach ihrer Meinung eine Dauerinnervation, die auf dem Wege der motorischen Nerven dem Muskel zufliesst, als Ursache der langdauernden tetanischen Starre auszuschliessen.

Bei dieser ganzen widerspruchsvollen Diskussion fällt auf, dass das Novokain zur Aufklärung der Natur der tetanischen Muskelstarre benutzt wird, ohne dass vorher seine Wirkung auf den innervierten und durchbluteten normalen Muskel des Warmblüters hinreichend untersucht wurde. Ehe man namentlich die zuletzt angeführten Befunde von Erich Meyer und Weiler und die gegenteiligen Ansichten von Fröhlich und H. Meyer gegeneinander abwägen kann, muss festgestellt werden, ob am nicht tetanusstarren normalen Muskel das Vermögen zur Dauerkontraktion (zentralbedingter Tonus) und zur aktiven Bewegung durch Novokain in verschiedener Weise beeinflusst wird. Hierbei muss vor allem die Wirkung der verschiedenen Novokaindosen quantitativ festgestellt werden. Wir haben deshalb zunächst bei Katzen im Zustand der Enthirnungsstarre geprüft, ob es gelingt, die Starre ganz oder teilweise zu lösen, ohne die Erregbarkeit vom. Nerven aus zu beeinträchtigen, und den Mechanismus einer derartigen Wirkung aufzuklären. Erst danach sind wir dazu übergegangen, entsprechende Versuche mit den gleichen Novokaindosen am tetanusstarren Muskel auszuführen, die uns dann auch zu einer ganz anderen Erklärungsweise des Einflusses von Novokain auf die Tetanusstarre geführt haben, als sie von den früheren Untersuchern angenommen wurde. Wir waren uns dabei von vornherein bewusst, dass die Enthirnungsstarre und die Tetanusstarre durchaus nicht wesensgleich zu sein brauchen, wie schon aus der Angabe von Fröhlich und H. Meyer ${ }^{1}$ ) und von Semerau und Weiler ${ }^{2}$ ) hervorgeht, dass der tetanusstarre Muskel stromlos sei.

\section{Die Wirkung des Novokains auf den Skelettmuskel im Zustand der Enthirnungsstarre.}

Die zu den Versuchen verwendeten Katzen wurden ätherisiert, die Karotiden abgebunden, die Vagi durchschnitten, das Rückenmark am zwölften Brustwirbel freigelegt, die Dezerebrierung zwischen vorderen und hinteren Vierbügeln ausgeführt, und das ganze Gehirn proximal vom Enthimungsschnitt ausgeräumt. Darauf wurde die Äthemarkose ab-

1) A. Fröhlich und H. H. Meyer, Untersuchungen über die Aktionsströme anhaltend verkürzter Muskeln. Zentralbl. f. Physiol. Bd. 26 S. 269. 1912. - Vgl. auch Munchner med. Wochenschr. 1917 S. 290.

2) M. Semerau und L. Weiler, Elektromyograph. Untersuchungen am tetanischkranken starren Muskel. Zentralbl.f. Physiol. Bd. 33 S. 69. 1918. 
gestellt und das Tier mit künstlicher Atmung liegen gelassen, bis sich eine gute Enthirnungsstarre ausgebildct hatte. Sämtliche Prüfungen wurden an den Vorderbeinen, und zwar am Triceps (Ellbogenstrecker) ausgeführt. War die Starre gering, so wurde sie durch Durchtrennung des Pückenmarks am zwölften Brustwirbel.(meist beträchtlich) verstärkt.

Das Novokain wurde stets in den Triceps der einen Seite injiziert, das andere Bein diente dann zum Vergleich. In den Triceps dieses Vergleichsbeines wurde in den meisten Versuchen eine ebenso grosse Menge physiologischer Kochsalzlösung eingespritzt; dieses erwies sich stets als wirkungslos. Bei der intramuskulären Injektion wurde immer sorgfältig darauf geachtet, dass die Spitze der Nadel sioh im Muskelbauche befand und nicht subkutan. lag. Wenn am Anfang des Versuches das eine Bein eine stärkere Enthirnungsstarre im Ellbogen zeigte als das andere, so wurde Novokain stets in das erstere injiziert. Gewöhnlich zeigten aber beide Beine gleiche Starre.

Als Maass der Starre und der Novokainwirkung wurde der Vergleich mit dem Bein der Gegenseite benutzt. Dabei wurde entweder das Tirr in Pü̈ckenlage gebracht, die beiden Oberarme parallel gestellt und nun der Winkel gemessen, den der Unterarm mit demsielben bildete. Oder es wurde der Winkel bestimmt, hei welchem zuerst bei passiver Beugung des Filbogens ein Widerstand fühlbar wurde. Oder es wurden beide Arme mit gleichmässigem Druck im Ellbogen gebeugt und der Winkel der beiden Ellbogen danach gemessen. Auf diose Weise war es möglich, ein möglichst objektives Maass der Enthirnungsstarre in den verschiedenen Stadien des Versuches zu gewinnen.

Ausserdem wurde vor und nach Novokain der Einfluss verschiedener Kopfstellungen auf den Tricepstonus ") untersucht. Bcim Vorhandensein von deutlichen tonischen Labyrinthreflexen tritt beim Umlegen des Tieres aus der Seiten- in Rückenlage eine starke Streckung beider Vorderbeine ein, deren Crad auf die oben angegebene Weise gemessen werden kann. Beim Kopfdrehen in Seitenlage reagiert vor allem das obere Bein (Streckung, wenn der Scheitel nach unten gedreht wird; Abnabme des Strecktonus, wenn der Scheitel nach oben gedreht wird); wenn die Labyrinthreflexe überwiegen, reagiert das unten liegende Vorderbein im gleichen Sinne, wenn die Halsreflexe überwiegen, dagegen im umgekehrten Sinne. Man tut daher gut, die Prüfung des Einflusses von Kopfdrehen in Seitenlage nacheinander in beiden Seitenlagen vorzunehmen.

In denjenigen Fällen, in welchen nach Novokaineinspritzung der Tricepstonus bei symmetrischer Kopfstellung in Seitenlage schon voll-

1) R. Magnus und A. de Kleyn, Die Abhängigkeit des Tonus der Extremitätenmuskeln von der Kopf́stellung. Pflüger's Archiv Bd. 145 S. 455.1912. 
ständig geschwunden ist, kann man ihn durch Verstärkung der tonischen Innervation durch Kopfdrehen oder durch Umlegen des Tieres in Rückenlage häufig noch zum Vorschein rufen und seine Intensität messen.

Zur Prüfung der aktiven Beweglichkeit diente entweder der grkreuzte Streckreflex, ausgelöst durch Kneifen der Pfote des Vorderbeines der Gegenseite. In einzelnen Fällen liess sich auch durch Beklopfen des Endgliedes des zu prüfenden Vorderbeines eine reflektorische Streckung des Ellbogens durch Tricepskontraktion hervorrufen. Dieser Reflex soll im folgenden als ,:Tricepsreflex" bezeichnet werden. Er ist beim dezerebrierten Tiere nur in Ausnahmefällen auszulösen, während er beim dekapitierten Tier gewöhnlich mit grösster Deutlichkeit nachweisbar ist. Manchmal gelingt es auch, nach dem Auslösen des gleichseitigen Beugereflexes (auf Kneifen der Pfote des Versuchsbeines) eine Rückstosskontraktion (,,Rebound-contraction " von Sherrington) hervorzurufen, welche dann ebenfalls als Beweis für das Vorhandensein zentraler motorischer Innervation benutzt werden kann.

Bei allen diesen Prüfungen muss man sorgfältig darauf achten, dass es sich im Einzelfalle wirklich um eine Kontraktion bzw. um Enthirnungsstarre im Triceps, d. h. in Ellbogen, handelt, und dass nicht etwa die beobachteten Bewegungen des Armes durch Kontraktion der Schultermuskeln hervorgerufen werden. Denn diese sind natürlich, wenn der Triceps durch Novokain bereits völlig gelähmt ist, noch unverändert erregbar.

Am Schluss des Versuches wurde dann in einer Reihe von Versuchen beiderseits der Plexus brachialis freigelegt und die indirekte Erregbarkeit des Triceps vom Nerven aus mit dem faradischen Strome geprüft. Zu diesem Zwecke wurden die Muskeln, welche das Schulterblatt mit dem Rumpfe verbinden, vom Rücken aus durchschnitten, der Brachialplexus sorgfältig vom umgebenden Bindegewebe gesäubert und der Erfolg oder Nichterfolg der Reizung durch Besichtigung des freigelegten Triceps kontrolliert. Zum Schlusse wurde beiderseits die direkte Erregbarkeit des Triceps gemessen. Zu den Versuchen diente ein nach Kronecker geeichtes Induktorium.

Das Ergebnis der Versuche mag durch einjge Protokolle veranschaulicht werden.

Versuch XV, am 10. Dezember 1918. Katze 1,28 kg. Äther, Karotiden abgebunden, Vagi durchtrennt, Freilegung des Rückenmarkes am zwölften Brustwirbel. Dezerebrierung mit Ausräumung des Grosshirns.

9 h $50^{\prime}$. Ende der Operation, Äther abgestellt. Gute Starre der vier Beine, Spontanatmung.

$10^{\text {h }} 40^{\prime}$. Stärkste Starre der vier Beine. Auf Kopfdrehen in Seitenlage starke Hals- und Labyrinthreflexe. Auf Umlegen in Rückenlage deutliche Labyrinthreflexe. In Seitenlage bei symmetrischer Kopf- 
stellung linkes Vorderbein maximal gestreckt, rechts Ellbogenwinkel $135^{\circ}$.

$10^{\mathrm{h}} 48^{\prime}$. In den linken Triceps wird $1 \mathrm{ccm} 1 \%$ iges Novokain, in den rechten $1 \mathrm{ccm} 0,9 \%$ iges $\mathrm{NaCl}$ eingespritzt.

$10^{\mathrm{h}} 54^{\prime}$. In Rückenlage Ellbogenwinkel links $110^{\circ}$, rechts $135^{\circ}$ (das vorher stärker gestreckte linke Vorderbein hat also jetzt geringeren Tricepstonus als das rechte).

$10^{\mathrm{h}} 56^{\prime}$. In Rückenlage Ellbogen links $90^{\circ}$, bietet geringen Widerstand gegen Bevgen, rechts $135^{\circ}$, bietet starken Widerstand gegen Beugen. Auf Kopfdrehen in rechter Seitenlage reagiert das linke Vorderbein stark (Scheitel unten maximale Streckung, Mittelstellung $90^{\circ}$, Scheitel oben $45^{\circ}$ ).

$11 \mathrm{~h} 00^{\prime} .1 / 2 \mathrm{ccm} 1 \%$ iges Novokain in den linken, $1 / 2 \mathrm{ccm} 0,9 \%$ iges $\mathrm{NaCl}$ in den rechten Triceps eingespritzt.

$11 \mathrm{k} 05^{\prime}$. In Seitenlage Ellbogen links $90^{\circ}$, geringer Widerstand beim Beugen, rechts $135^{\circ}$, starker Widerstand beim Beugen. Auf Kopfdrehen in Seitenlage starke Reaktion des linken Vorderbeines im Ellbogen, wie 10 h $56^{\prime}$.

$11^{\text {h }} 12^{\prime}$. In rechter Seitenlage bei symmetrischer Kopfstellung Ellbogen links $45^{\circ}$, rechts $185^{\circ}$. Bei Kopfdrehen mit Scheitel unten Ellbogen links $90^{\circ}$, bei Scheitel oben $0^{\prime \prime}$.

Darauf Freilegung des Plexus brachialis und des Triceps beiderseits. Faradische Reizung, zwei Akkumulatoren, kein Extrawiderstand im sekundären Kreis.

Erregbarkeit rechts (Normalseite): indirekt $20 \mathrm{Kronecker,}$ direkt 200

Erregbarkeit links (Novokainseite): indirekt 15 direkt 150

Ergebnis: Nach Einspritzung von 1 und von $1 \frac{1}{2} \mathrm{ccm}$ Novokain in den Triceps erfolgt eine sehr starke Abnahme der hochgradigen Streckstarre. Reaktion auf Kopfdrehen erhalten, führt aber zu geringeren Tonusgraden als vorher. Indirekte und direkte Erregbarkeit des Triceps vollständig unvermindert.

Versuch XI, am 2. Dezember 1918. Katze 1,3 kg. Äther, Karotiden abgebunden, Vagi durchtrennt. Dezerebrierung mit Ausräumung der vorderen Schädelgrube.

$6 \mathrm{~h} 55^{\prime}$. Ende der Operation. Gute Starre.

$7 \mathrm{~h} \mathrm{25}$. Deutliche Starre der $\nabla$ orderbeine, links etwas kräftiger als rechts. In Ruckenlage rechter Ellbogen $90^{\circ}$, linker $130^{\circ}$. In Seitenlage gute Reaktion beiderseits auf Kopfdrehen.

$7 \mathrm{~h} 30^{\prime}$. Kräftige Starre der Vorderbeine, links stärker als rechts.

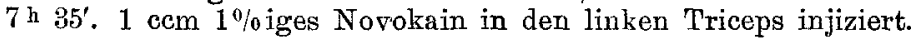

7 h $38-42$ '. Tonusunterschied der beiden Vorderbeine ver. schwunden. Deutliche Reaktion beider Vorderbeine auf Kopfdrehen in Seitenlage.

$7 \mathrm{~h} 44^{\prime} .1 / 2 \mathrm{~cm} 1 \%$ iges Novokain in den linken Triceps eingespritzt.

7 b $54^{\prime}$. Starre beiderseits gleich. Iinkes Bein reagiert noch deutlich auf Kopfbewegungen, wenn auch schwächer als das rechte. 1/2 ccm $1 \%$ iges Novokain in den linken Triceps.

$8 \mathrm{~h} 00^{\prime}$. Starre im linken Ellbogen geringer als im rechten, aber immer noch kräftig. Das linke Vorderbein zeigt noch schwache Reaktion auf Kopfdrehen.

8 h $01^{\prime}$. 1/2 ccm 1\% iges Novokain in den linken Triceps. 
8 b 07 '. Starre im linken Triceps sehr gering (hat auch im rechten Triceps abgenommen). Tricepsreflex auf Beklopfen der Pfote links deutlich, rechts kräftig. Auf Kopfdrehen keine Reaktion des linken Triceps, deutliche Reaktion des rechten Triceps.

Beiderseits Freilegung des Plexus brachialis und Triceps. Faradische Reizung, zwei Akkumulatoren, kein Extrawiderstand im sekundären Kreis.

Erregbarkeit rechts (Normalseite): indirekt 50-60 Kronecker, direkt 425

Erregbarkeit links (Novokainseite): indirekt 60 direkt 825

Ergebnis: Nach $1 \mathrm{ccm} 1 \%$ igem Novokain beginnt die Starre abzunehmen, nach $2 \frac{1}{2} \mathrm{ccm}$ ist sie fast ganz geschwunden. Reaktion auf Kopfdrehen aufgehoben. Tricepsreflex dagegen deutlich. Indirekte Erregbarkeit unverändert erhalten.

Versuch XII, am 6. Dezember 1918. Katze 1,15 kg. Äther, Karotiden abgebunden, Vagi durchtrennt. Freilegung des Rückenmarks am zwölften Brustwirbel. Dezerebrieren mit Ausräumung der vorderen Schädelgrube. $10^{\text {h }} 30^{\prime}$. Ende der Operation. Starre der Vorderbeine.

11 h 08'. Durchtrennung des Ruckenmarks am zwölften Brustwirbel. Enorme Starre der Vorderbeine und des Nackens. Beugung der Vorderbeine kaum möglich. Kopfdrehen in Seitenlage bewirkt Labyrinthreflexe.

11 h $13 \%$. 1 com 1\%iges Novokain in den linken, $1 \mathrm{com} 0,9 \%$ iges $\mathrm{NaCl}$ in den rechten Triceps.

$11^{\text {h }} 18^{\prime}$. In Seitenlage mit symmetrischer Kopfstellung Ell bogen links $90^{\circ}$, rechts $135^{\circ}$. Auf Kopfdrehen mit Schejtel unten maximale Starre beider Vorderbeine. Gekreuzter Streckreflex auf das linke Bein deutlich.

11 h 25'. $1 \mathrm{ccm} 1 \%$ iges Novokain in den linken, $1 \mathrm{ccm} 0,9 \%$ iges $\mathrm{NaCl}$ in den rechten Triceps. (Die Novokaineinspritzung ist wahrscheinlich nicht in, sondern neben den Muskel erfolgt.)

11 h $30^{\prime}$. In Rückenlage Starre rechts maximal, links sehr gering. In rechter Seitenlage Starre rechts $90^{\circ}$, links $0^{\circ}$. Auf Kopfdrehen mit dem Scheitel nach unten rechts Starre maximal, links deutlich geringer. Gekreuzter Streckreflex auf das linke Vorderbein (Ellbogen) deutlich.

$11 \mathrm{~h} 39^{\prime} .{ }^{1 / 2} \mathrm{ccm} 1 \%$ iges Novokain links, $1 / 2 \mathrm{ccm} 0,9 \%$ iges $\mathrm{NaCl}$ rechts.

11 h $46^{\prime}$. In Seitenlage Starre.links noch nicht ganz geschwunden, aber viel geringer als rechts. Starke Reaktion beiderseits auf.Kopfdrehen, aber auch bei Scheitel unten ist die Starre links viel geringer als rechts. Streckreflex links positiv.

$11 \mathrm{~h} 52$. $1 \mathrm{ccm} 1 \%$ iges Novokain in den linken, $1 \mathrm{ccm} 0,9 \%$ iges $\mathrm{NaCl}$ in den rechten Triceps.

11 h 58'. Starre links noch nicht vollständig geschwunden. Linkes Bein wird gestreckt gehalten, lässt sich aber total beugen, während rechts bei gleichem Druck der Ellbogen einen Winkel von $135^{\circ}$ bildet. Deutliche Reaktion auf Kopfdrehen in Seitenlage.

12 h 03 . $1 \mathrm{ccm} 1 \%$ iges Novokain links, $1 \mathrm{ccm} 0,9 \%$ iges $\mathrm{NaCl}$ rechts.

1) Die einzelnen Teile der Tricepsoberfläche sind immer sehr verschieden empfindlich gegen den faradischen Strom, so dass bei der Ermittelung der direkten Muskelerregbarkeit auf kleine Unterschiede kein Wert gelegt werden darf. 
12 h $08^{\prime}$. Im linken Vorderbein ist noch immer etwas Starre vorbanden, deutliche Reaktion auf Kopfdrehen, deutlicher gekreuzter Streckreflex.

12 h 12'. $1 \mathrm{ccm} 1 \%$ iges Novokain links, $1 \mathrm{ccm} 0,9 \%$ iges $\mathrm{NaCl}$ rechts.

12 i $17^{\prime}$. Zustand unverändert.

$12 \mathrm{~h} \mathrm{21}$. $1 \mathrm{ccm} 1 \%$ iges Novokain links, $1 \mathrm{ccm} 0,9 \%$ iges $\mathrm{NaCl}$ rechts.

12 h $26^{\prime}$. In Rückenlage Tonus im linken Triceps verschwunden (bei deutlichem Tonus:der linken Schultermuskeln), im rechten Triceps stark. Reaktion auf Kopfdrehen in Seitenlage links minimal (aber. noch deutlich), rechts sehr stark. Gekreuzter Streckreflex auf das linke Vorderbein verschwunden.

$12 \mathrm{~h} 31^{\prime}$. $1 / 2 \mathrm{ccm} 1 \%$ iges Novokain links, $1 / 2 \mathrm{ccm} 1 \%$ iges $\mathrm{NaCl}$ rechts.

12 h 37'. Starre im linken Ellbogen verschwunden, rechts sehr stark. Auf Kopfdrehen in Seitenlage reagiert der linke Triceps höchstens minimal, die linke Schulter deutlich; der rechte Triceps maximal. Gekreuzter Streckreflex verschwunden.

Beiderseits wird der Plexus brachialis und der Triceps freigelegt. Faradische Reizung, zwei Akkumulatoren, kein Extrawiderstand.

Erregbarkeit rechts (Normalseite): indirekt $400 \mathrm{Kronecker}$ direkt 1500

Erregbarkeit links (Novokainseite): indirekt 3000 unwirksam, direkt 2000 wirksam.

Ergebnis: Sehr starke Starre. Diese wird durch 1 ccm 1\%iges Novokain bereits deutlich vermindert, während die Reaktion auf Kopfdrehen und der gekreuzte Streckreflex erhalten bleiben. Auf $2 \mathrm{ccm}$ ist die Starre minimal, die Reaktion auf Kopfdrehen und der Streckreflex deutlich vorhanden. Dieser Zustand ist auch nach $5 \frac{1}{1 / 2} \mathrm{ccm}$ noch unverändert erhalten. Erst auf $6 \frac{1 / 2}{2} \mathrm{~cm}$ verschwindet der gekreuzte Streckreflex, auf $7 \mathrm{ccm}$ die Reaktion auf Kopfdrehen. Die indirekte Erregbarkeit des linken Triceps ist erloschen, die direkte fast unvermindert erhalten.

Diese Versuche zeigen, und alle anderen Experimente haben es bestätigt, dass kleine Novokaindosen, intramuskulär eingespritzt, die Enthirnungsstarre sehr beträchtlich vermindern, ohne sie in den meisten Fällen vollständig aufzuheben. In diesem Zustand ist aber die aktive Beweglichkeit noch unverändert erhalten, und bei indirekter faradischer Reizung vom Plexus aus findet man dieselben Schwellenwerte wie am unvergifteten Muskel. Erst be trächtlich grössere Dosen Novokain heben die indirekte Erregbarkeit des Muskels und damit natürlich auch die letzten Reste von Enthirnungsstarre und die aktive Beweglichkeit auf. Die direkte faradische Erregbarkeit ist dann aber noch erhalten und häufig kaum vermindert.

Im einzelnen haben sich folgende Verhältnisse ergeben. Einspritzung von $1 / 4 \mathrm{cem} 1 \%$ igem Novokain in den Triceps erwies sich als wirkungslos. Die kleinste Dose, welche eine Herabsetzung der Enthirnungs- 
starre bewirkte, war $1 / 2 \mathrm{ccm}$. Unter sieben Versuchen war eine Dosis von 1 ecm nur einmal nicht genügend, die Enthirnungsstarre zu vermindern, so dass $2 \mathrm{ccm}$ eingespritzt werden mussten. (Das Gewicht der Versuchstiere war meist zwischen $I$ und $1,3 \mathrm{~kg}$. Von einer Berechnung der Dosen auf $1 \mathrm{~kg}$ Körpergewicht wird Abstand genommen, weil eigentlich nur das Gewicht des injizierten. Triceps in Betracht kommt, der auch bei Tieren gleichen Gewichtes nicht gleich schwer ist.) Während die Herabsetzung der Enthirnungsstarre nach der Injektion der genannten kleinen Dosen in allen Fällen sehr deutlich und. unzweifelhaft ist, ist der Grad der Starre, welcher dann noch zurückbleibt, in den verschiedenen Versuchen sehr wechselnd. War die Starre vorher gering, so kann sie nach Novokain fast vollständig schwinden, so dass sie kaum noch nachweisbar bleibt. Handelt es sich dagegen um ein Tier mit beträchtlichem Tricepstonus, so kann auch nach der Injektion noch eine deutliche Starre zurückbleiben, die allerdings dann immer sehr viel geringer ist als vorher. Die Gründe für dieses wechselnde Verhalten können erst weiter unten' auseinandergesetzt werden. Auch wenn nach den kleinen Novokaindosen die Enthirnungsstarre fast vollständig geschwunden ist, kann man meist durch geeignete Lagerung des Kopfes (Rückenlage, Kopfdrehen in Seitenlage) die tonischen Hals- und Labyrinthreflexe auf die Gliedermuskeln zum Vorschein rufen und dadurch den Tonus des schlaffen Triceps wieder steigern. Der Grad, bis zu welchem dieses möglich ist, hängt, solange die indirekte Erregbarkeit des Triceps nicht abgenommen hat, davon $a b$, wie stark diese Reflexe bei dem betreffenden Versuchstier ausgesprochen sind, was von Fall zu Fall wechselt und am Beginn jedes Versuches festgestellt werden muss.

Zur völligen Aufhebung der indirekten Muskelerregbarkeit vom Plexus aus sind bei Tieren von $1-1,3 \mathrm{~kg} 7-8 \mathrm{~cm} 1 \%$ iges Novokain erforderlich. In einem Falle war bei einem Tiere von $1,5 \mathrm{~kg}$ nach $8 \mathrm{~cm}$ noch eine geringe Spur von Erregbarkeit vorhanden (4000 Kronecker gegenüber $20 \mathrm{Kr}$. auf der Normalseite). Andererseits war einmal bei einer Katze von 1,9 kg nach $4 \mathrm{ccm} 1 \%$ igem Novokain die indirekte Erregbarkeit bereits erloschen.

Die kleinsten Dosen, bei welchen die indirekte Erregbarkeit abnahm, ohne jedoch vollständig zu erlöschen, waren $3 \mathrm{ccm}$ bei einer Katze von $1,9 \mathrm{~kg}$ (2000 Kronecker gegen $100 \mathrm{Kr}$. auf der Normalseite) und $2 \mathrm{ccm}$ bei einem Tiere von $0,75 \mathrm{~kg}(1000 \mathrm{Kr}$. gegen $100 \mathrm{Kr}$. auf der Normalseite). In dem letzteren Falle war noch eine schwache Reaktion des betreffenden Triceps auf Kopfdrehen und beim gekreuzten Streckreflex vorhanden. Andererseits war in einem Versuche bei einem Tiere von $1,5 \mathrm{~kg}$ nach $8 \mathrm{ccm}$ Novokain noch eine schwache Reaktion bei Plexusreizung erhalten ( $3000 \mathrm{Kr}$. gegen $20 \mathrm{Kr}$. auf der Normalseite). 
Wenn also auch im Einzelfalle die Dosen etwas wechseln, so ist doch die wesentliche Tatsache über jeden Zweifel erhaben, dass nach kleinen Novokaindosen die Starre beträchtlich abnimmt, ohne dass die aktive Beweglichkeit und die indirekte Erregbarkeit vermindert ist, und dass erst beträchtlich grössere Novokaindosen erforderlich sind, um aktive Beweglichkeit und indirekte Erregbarkeit aufzuheben. Damit ist für die Enthirnungsstarre der Befund, den Erich Neyer und Weiler am tetanusstarren Muskel des Menschen erhoben haben, vollinhaltlich bestätigt.

Es fragt sich nun, wie diese Tatsache zu erklären ist. Dazu ist es nötig, die Entstehung der Enthirnungsstarre zu erörtern. Durch Sherrington ${ }^{1}$ ) ist nachgewiesen worden, dass dieselbe ein tonischer Reflex ist, welcher hauptsächlich durch proprioceptive Erregungen zustande kommt, die in den tonisch kontrahierten Muskeln selber ihren Ursprung nehmen. Ausser dieser Hauptquelle spielen aber noch andere Dauererregungen eine Rolle. Zunächst proprioceptive Erregungen von anderen Muskeln desselben Gliedes und des Gliedes der Gegenseite, ferner Impulse von Hautnerven der Extremität, tonische Einflüsse vom Hals und den Labyrinthen und ausserdem noch von den anderen Körperregionen ${ }^{2}$ ). Schematisch können wir also sagen, dass die Enthirnungsstarre entsteht erstens auf Grund von proprioceptiven Erregungen, die von dem tonisch kontrahierten Muskel selbst ausgehen, und zweitens auf Grund von einer. grossen Gruppe von anderen afferenten Impulsen. Die proprioceptiven Erregungen, welche vom betreffenden Muskel selber ausgehen, spielen für die Enthirnungsstarre in allen Fällen eine sehr grosse Rolle. Die Impulse von den anderen Quellen addieren sich je nach der Lage des Einzelfalles in verschiedenem Grade hinzu.

Die in unseren Versuchen beobachtete Wirkung des Novokains auf den Skelettmuskel wird demnach verständlich, wenn man annimmt, dass kleine Dosen nach intramuskulärer Einspritzung ausschliesslich die sensiblen Nervenenden im Muskel selber lähmen. Dann werden die proprioceptiven Erregungen, welche von diesem Muskel selber ausgehen, aufgehoben und damit die wichtigste Quelle der Enthirnungsstarre beseitigt. Die Folge ist, dass die Starre abnimmt. Aber sie braucht nicht ganz zu schwinden, weil sensible Erregungen von anderen Körperteilen noch einwirken können. Vor

1) C. S. Sherrington, Integrative action of the nervous system p. 338. 1906. - Flexion reflex of the limb etc. Journ. of Physiol. vol. 40 p. 28. 1910.

2) R. Magnus und W. Storm van Leeuwen, Akute und dauernde Folgen des Ausfalles der tonischen Hals- und Labyrinthreflexe. Pflu ger's Archiv Bd. 159 S. 213 tf. 1914. 
allem ist das der Fall für die Erregungen vom Hals und den Laby. .rinthen, welche je nach der Erregbarkeit des Tieres ihre Wirkung noch entfalten können. In diesem Zustand ist die aktive Beweglichkeit vom Zentralnervensystem aus und die indirekte Erregbarkeit vom Plexus aus noch unvermindert erhalten. Erst sehr viel grössere Dosen lähmen die motorischen Nervenenden im Muskel und heben dadurch aktive Beweglichkeit und indirekte Erregbarkeit auf. Durch die von uns verwendeten Dosen tritt eine Lähmung des Muskels selber dagègen nicht ein.

Diese Hypothese ist experimenteller Prüfung zugänglich. Schaltet man die Sensibilität des zum Versuche benutzten Muskels durch Durchschneidung der zugehörigen Hinterwurzeln aus und erzeugt danach durch Dezerebrieren eine Enthirnungsstarre, so muss Einspritzung von kleinen Dosen Novokain ohne jeden Einfluss auf die Starre sein, während natürlich die grossen Dosen durch Lähmung der motorischen Nervenenden Starre, aktive Beweglichkeit und indirekte Erregbarkeit aufheben. Die Versuche haben ergeben, dass dieses tatsächlich der Fall.

Von vornherein war es nicht sicher. dass diese Experimente zum gewünschten Ziele führen würden, weil nach Hinterwurzeldurchschneidung die Starre der zugehörigen Muskeln gering zu sein pflegt.' Wenn man den Einfluss von Novokain untersuchen will, muss man aber an Muskeln arbeiten, wẹlche zu Anfang eine deutliche tonische Kontraktion zeigen: Es ist nun möglich gewesen, durch Durchschneidung des Rückenmarkes im untersten Brustterl und durch geeignete Lagerung des Kopfes bei den Tieren hinreichend starke Starre des Triceps zu erzielen.

Folgendes Versuchsbeispiel möge das Ergebnis veranschaulichen:

Versuch XVI, am 11. Dezember 1918. Katze 1,27 kg. Äther, Karotiden abgebunden, Vagi durchtrennt, Freilegung des Rückenmarks am zwölften Brustwirbel. Beiderseits werden die Hinterwurzeln extradural vom sechsten Halssegment bis zum zweiten Brustsegment durchtrennt ${ }^{1}$ ), Vorderwurzeln intakt gelassen. Dezerebrierung mit Ausräumen der vorderen Schädelgrube. $10^{\mathrm{h}} 25^{\prime}$. Ende der Operation, Tier schlaff.

$10^{\mathrm{h}} 55^{\prime}$. Gute Starre der Hinterbeine, Vorderbeine schlaff. Durchtrennung des Rückenmarks am zwölften Brustwirbel. Darauf sofort kräftige Starre der Vorderbeine, welche auf Kopfdrehen in Seitenlage gute Hals- und Labyrinthreflexe zeigen.

$11^{\text {h }}$ 04-11'. In Seitenlage mit symmetrischer Kopfstellung hat das linke Bein deutlichen Tonus $\left(90^{\circ}\right)$, das rechte $\nabla$ orderbein ist schlaff. Auf Kopfdrehen mit Schädel unten bekommt bei rechter Seitenlage das linke Vorderbein eine Starre von $135^{\circ}$, in linker Seitenlage das rechte Vorderbein $90^{\circ}$. Das linke Vorderbein hat also stärkere Starre als das rechte.

1) Der Triceps wird bei der Katze von $C_{s}$ und $T h_{1}$ innerviert. $S$. $u$. S. 198 und Polimanti, zit. nach v. R ynberk, Erg. d. Anatomie 18. 733.1908. 
11 h $13^{\prime} .1^{1 / 2} \mathrm{ccm} 1 \%$ iges Novokain in den linken, $1 \frac{1 / 2}{\mathrm{ccm}} 0,9 \%$ iges $\mathrm{NaCl}$ in den rechten Triceps.

$11 \mathrm{~h} 23^{\prime} .1 / 2 \mathrm{ccm} 1 \%$ iges Novokain in den linken, 1/2 ccm $0,9 \%$ iges $\mathrm{NaCl}$ in den rechten Triceps.

$11^{\mathrm{h}} 33^{\prime}$. 1/2 ccm 1\% iges Novokain in den linken, $1 / 2 \mathrm{ccm} 0,9 \%$ iges $\mathrm{NaCl}$ in den rechten Triceps.

$11 \mathrm{~h} 44^{\prime} .1 / 2 \mathrm{ccm} 1 \%$ iges Novokain in den linken, $1 / 2 \mathrm{ccm} 0,9 \%$ iges $\mathrm{NaCl}$ in den rechten Triceps.

$11^{\mathrm{h}} 51^{\prime} .1 \mathrm{ccm} 1 \%$ iges Novokain in den linken, $1 \mathrm{ccm} 0,9 \%$ iges $\mathrm{NaCl}$ in den, rechten Triceps.

$11 \mathrm{~h} 59^{\prime} .1 \mathrm{ccm} \mathrm{1 \%} \%$ iges Novokain in den linken, $1 \mathrm{ccm} 0,9 \%$ iges $\mathrm{NaCl}$ in den rechten Triceps.

$12 \mathrm{~h} 08^{\prime} .1 \mathrm{ccm} 1 \%$ iges Novokain in den linken, $1 \mathrm{ccm} 0,9 \%$ iges $\mathrm{NaCl}$ in den rechten Triceps.

12 h 13'. Nach Finspritzung von $6 \mathrm{ccm}$ Novokain in den linken Triceps ist der Zustand vollständig ungeändert. Der linke Triceps hat immer: noch mehr Tonus als der rechte. Derselbe nimmt in Rückenlage und in rechter Seitenlage mit Scheitel unten auf $135^{\circ} \mathrm{zu}$.

$12 \mathrm{~h} 15 \%$ ccm 1\%iges Novokain in den linken, $1 \mathrm{ccm} 0,9 \%$ iges $\mathrm{NaCl}$ in den rechten Triceps.

$12 \mathrm{~h} \mathrm{21}$. Der Strecktonus im linken Ellbogen hat etwas abgenommen, ist aber immer noch stärker als im rechten $\left(90^{\circ}\right.$ in rechter Seitenlage mit Scheitel unten).

$12 \mathrm{~h} \mathrm{25'.} 1 \mathrm{ccm} \mathrm{1 \%}$ iges Novokain in den linken, $1 \mathrm{ccm} 0,9 \%$ iges $\mathrm{NaCl}$ in den rechten Triceps.

$12^{h} 30^{\prime}$. Der Tonus im linken Ellbogen hat weiter abgenommen, ist aber noch nicht ganz geschwunden.

$12 \mathrm{~h} 32 \%$. $1 \mathrm{ccm} 1 \%$ iges Novokain in den linken, $1 \mathrm{~cm} 0,9 \%$ iges $\mathrm{NaCl}$ in den rechten Triceps.

$12^{\mathrm{h}} 38^{\prime}$. In Rückenlage deutlicher Tonus im rechten Ellbogen, keine Spur von Tonus im linken Ellbogen. Auf Kopfdrehen in rechter Seitenlage zeigt der linke Ėllbogen noch eine ganz minimale Reaktion.

Beiderseits Freilegung des Plexus brachialis und des Triceps. Faradische Reizung, zwei Akkumulatoren, kein Extrawiderstand. im sekundären Kreis.

Erregbarkeit rechts indirekt $200 \mathrm{Kr}$. direkt $600 \quad \mathrm{Kr}$. " links " 2000 " negativ, "600-800"

Sektion: Beiderseits Hinterwurzeln von $\mathrm{C}_{6}$ bis $\mathrm{Th}_{2}$ durehtrennt, alle Vorderwurzeln intakt.

Ergebnis : Nach Durchtrennung der Hinterwurzeln für den Triceps tritt genügende Enthirnungsstarre auf. Intramuskuläre Ninspritzung von kleinen Novokaindosen ist ohne jeden Einfluss a uf die Starre. Selbst nach $6 \mathrm{ccm} 1 \%$ igem Novokain ändert sich dieselbe nicht. Erst $7 \mathrm{ccm}$ vermindert die Starre, $8 \mathrm{ccm}$ hebt sie auf. Danach ist die indirekte Erregbarkeit erloschen, die direkte fast unvermindert erhalten.

Nachdem dieser Versuch der Erwartung vollständig entsprochen hatte, dass nach Ausschaltung der sensiblen Nerven kleine Novokaindosen ohne jeden Einfluss auf die Enthimungsstarre sind und erst grosse Dosen, welche die motorischen Nervenenden im Muskel lähmen, die Starre aufheben, war noch festzustellen, ob tatsächlich sich nach 
den kleinsten Novokaindosen, welche gerade eben die Starre vermindern, auch bereits eine Herabsetzung der indirekten Erregbarkeit des desensibilisierten Muskels nachweisen lässt. Auch dieses ișt der Fall, wie nachfolgender Versuch zeigt.

Versuch XX, am 18. Dezember 1918. Katze 1,5 kg. Äther, Karotiden abgebunden, Vagi durchtrennt. Freilegung des Rückenmarkes am zwölften Brustwirbel. Extradurale Durchschneidung der Hinterwurzeln links von $\mathrm{C}_{5}$ bis $\mathrm{Th}_{2}$, rechts von $\mathrm{C}_{6}$ bis $\mathrm{Th}_{2}$. (Ausserdem wird, wie die später vorgenommene Sektion zeigte, links noch die Vorderwurzel von $\mathrm{Th}_{2}$ durchschnitten, welche sich aber an der Innervation des Triceps nicht mehr beteiligt.) Dezerebrierung mit Ausräumen der vorderen Schädelgrube. Operationsdauer 87 Minuten.

$10^{\mathrm{h}} 02^{\prime}$. Ende der Operation. Geringer Tonus im linken Triceps. Spontane Atmung.

$10^{\mathrm{h}} 40^{\prime}$. In Rückenlage Tricepstonus links $135^{\circ}$, rechts $90^{\circ}$. Auf Kopfdrehen in Seitenlage sehr starke Reaktion des linken, keine Reaktion des rechten Ellbogens.

$10^{\mathrm{h}}$ 45'. Durchtrennung des Rückenmarkes am zwölften Brustwirbel. Zunahme der Starre der Vorderbeine. In Rückenlage maximale Streckung beider Vorderbeine; das linke ist vollständig steif, das rechte bietet gegen passive Beugung etwas weniger Widerstand. Kopfdrehen in Seitenlage macht starke Labyrinthreflexe an beiden Vorderbeinen. Da das linke Vorderbein den stärkeren Strecktonus hat, wird in dieses Novokain eingespritzt.

Von $10^{\mathrm{h}} 54^{\prime}$ bis $11^{\mathrm{h}} 47^{\prime}$ werden im ganzen $7 \mathrm{ccm} 1 \%$ iges Novokain in den linken und $7 \mathrm{ecm} 0,9 \%$ iges $\mathrm{NaCl}$ in den rechten Triceps eingespritzt, ohne dass sich der Zustand ändert. Noch immer hat das linke Bein stärkeren Strecktonus im Ellbogen als das rechte. In Rückenlage erfolgt deutliche Tonuszunahme beider Beine, links aber stärker als rechts. Auf Kopfdrehen in Seitenlage reagieren beide Vorderbeine kräftig.

$11^{\mathrm{h}} 54^{\prime} .1 \mathrm{ccm} 1 \%$ iges Novokain in den linken, $1 \mathrm{ccm} 0,9 \%$ iges $\mathrm{NaCl}$ in den rechten Triceps.

$12^{\mathrm{h}}$ 04'. Das linke Vorderbein reagiert noch auf Kopfdrehen in Seitenlage, hat aber in Rückenlage deutlich weniger Strecktonus im Ellbogen als das rechte.

Freilegung des Plexus brachialis und Triceps beiderseits. Faradische Reizung, ein Akkumulator, kein Extrawiderstand.

Erregbarkeit rechts indirekt $20 \mathrm{Kr}$., direkt $250-500 \mathrm{Kr}$.

$$
\text { " links " } 3000 " \text { " 300-350" }
$$

Ergebnis: Nach Denervierung des Triceps ist Einspritzung von $7 \mathrm{ccm} 1 \%$ igem Novokain ohne jede Wirkung auf die Enthirnungsstarre. Nach $8 \mathrm{ccm}$ beginnt die Starre nachzulassen. Dabei ist die indirekte Erregbarkeit vom Nerven aus beträchtlich herabgesetzt, die direkte praktisch ungeändert.

In demselben Sinne fielen zwei weitere Versuche aus. In dem einen war bei einer Katze von 1,15 kg die Starre im desensibilisierten Triceps $\left(\mathrm{C}_{6}-\mathrm{Th}_{2}\right)$ nach $3 \mathrm{ccm} 1 \%$ igem Novokain noch unvermindert und begann nach $4 \mathrm{ccm}$ abzunehmen; dabei war die indirekte Erregbarkeit von $20 \mathrm{Kr}$. auf der Kontrollseite bis auf $2000 \mathrm{Kr}$. auf der 
Novokainseite herabgesetzt. In dem anderen Versuche war bei einer Katze von $2,65 \mathrm{~kg}$ die Starre im desensibilisierten Triceps $\left(\mathrm{C}_{5}-\mathrm{Th}_{2}\right)$ nach $4 \mathrm{ccm} 1 \%$ igem Novokain ganz unvermindert, nach $5 \mathrm{ccm}$ war eine ganz unsichere und. zweifelhafte Andeutung von Tonusabnahme vorhanden, nach $6 \mathrm{ccm}$ wurde eine geringe Abnahme deutlich. Der Triceps hatte aber noch Enthirnungsstarre und zeigte Hals- und Labyrinthreflexe. Die indirekte Erregbarkeit war von 20 (Normalseite) bis auf $4000 \mathrm{Kr}$. (Novokainseite) herabgesetzt ${ }^{1}$ ).

Diesen vier positiven Versuchen, welshe übereinstimmend zeigen, dass nach Durchtrennung der zugehörigen Hinterwurzeln kleine Novokaindosen ohne Wirkung auf die Enthirnungsstarre sind, und dass erst solche Dosen die Starre vermindern bzw. aufheben, welche die motorischen Nervenenden im Muskel lähmen, steht ein Versuch gegenüber, der im umgekehrten Sinne ausfiel. Hier war bei einem Tier von $1,15 \mathrm{~kg}$ nach $31 / \mathrm{ccm} 1 \%$ igem Novokain eine geringe Abnahme des Tricepstonus eingetreten, während die indirekte Erregbarkeit sich nicht geändert hatte (beiderseits $15 \mathrm{Kr}$.). Bei dieser Katze hatte der Tonus des Versuchsmuskels schon vor der Novokaininjektion eine 'Tendenz zur Abnahme gezeigt. Da es in Experimenten an dezerebrierten Tieren nicht selten vorkommt, dass die Starre in einem oder in beiden Beinen ohne direkt erkennbare Ursache abnimmt, sind wir berechtigt, das Ergebnis dieses Versuches als Zufallsresultat und nicht als Folge der Norokaininjektion aufzufassen, um so mehr, als die übrigen vier Versuche durehaus übereinstimmend und positiv ausfielen.

Die Versuche haben somit zu folgendem Ergebnis geführt:

Novokain in kleinen Dosen (im Mittel $1 \mathrm{ccm} 1 \%$ bei Tieren v,on etwa $1 \mathrm{~kg}$ ) in den Triceps dezerebrierter Katzen eingespritzt, lähmt die proprioceptiven sensiblen Muskelnerven und vermindert dadurch die (reflektorisch bedingte) Enthirnungsstarre. Bis zu welchem Grade dieselbe abnimmt, hängt davon ab, in welchem Ausmaasse sich im Einzelfalle noch andere reflektorische Einflüsse am $\mathrm{Zu}$ standekommen der Starre des betreffenden Triceps beteiligen. In diesem Stadium lassen sich durch Vermittelung des Zentralnervensystems noch kräftige Reflexe auf den Triceps hervorrufen. Bei faradischer Reizung des Plexus brachialis zeigt sich die indirekte Erregbarkeit quantitativ unvermindert.

1) In diesem letzteren Versuch war die Starre der Vorderbeine auch nach Durchtrennung des Rückenmarkes am zwölften Brustwirbel zunächst zu gering, um die Wirkung von Novokain untersuchen zu können. Es wurde daher $0,08 \mathrm{mg}$ Strychninnitrat intravenös eingespritzt. Diese Dosis steigert nach den Erfahrungen von Magnus und Wolf (P fllüger's Arch. Bd. 149 S. 447. 1913) die Enthirnungsstarre beträchtlich, ohne die Reflexerregbarkeit störend zu erhöhen. Ausserdem nehmen die tonischen Hals- und Labyrinthreflexe stark zu, ändern aber ihren Charakter nicht. Dieser Erfolg trat auch in dem geschilderten $\nabla$ ersuch mit grösster Deutlichkeit ein, so dass jetzt die Novokainwirkung untersucht werden konnte. 
Grosse Dosen Novokain (in den meisten Fällen 6-8 cem 1\% bei Katzen ron etwa $1 \mathrm{~kg}$ ) lähmen auch die motorischen Nervenenden im. Muskel und heben damit die Enthirnungsstarre völlig auf. Reflexe auf den Triceps sind nicht mehr auszulösen, die indirekte Erregbarkeit erlischt. Dagegen ist der Muskel selbst (meist unvermindert) noch direkt erregbar.

\section{Die Wirkung kleiner Novokaindosen auf den Skelettmuskel normaler Katzen.}

Die bisher geschilderten Versuche gaben zu der Frage Anlass, ob es auch bei normalen, nicht dezerebrierten Tieren gelingt, durch kleine Novokaindosen die proprioceptive sensible Innervation eines bestimmten Muskels vorübergehend bei erhaltener aktiver Beweglichkeit auszuschalten und die Ausfallserscheinungen zu studieren. Auch diese Experimente wurden hauptsächlich am Triceps der Katze angestellt.

Spritzt man einer Katze in den Triceps des einen Vorderbeines $0,8-1,5 \mathrm{ccm}$, im Mittel $1 \mathrm{ccm}$ pro Kilogramm einer $1 \%$ igen Lösung von Novokain $-\mathrm{HCl}$, so entwickelt sich nach etwa 5 Minuten eine ganz auffallende Störung. Der Strecktonus des Ellbogens der injizierten Seite nimmt beträchtlich ab oder schwindet gänzlich, so dass passive Beugung des Unterarmes auf keinen Widerstand mehr stösst. Steht das Tier auf dem Boden, so kann man auf das Schulterblatt der Normalseite einen sehr kräftigen Druck ausüben, ohne dass das Tier einknickt, während bei Druck auf das Schulterblatt der injizierten Seite das Vorderbein. sofort nachgibt. Packt man das Tier am Becken und Nacken und lässt es allein mit dem normalen Vorderbein auf dem Tische stehen, so trägt dieses Vorderbein den Körper, während derselbe Versuch mit dem Vorderbein der injizierten Seite nicht gelingt und der Vorderkörper des Tieres auf den Tisch fällt, weil der Ellbogen widerstandslos nachgibt. Wenn das Tier läuft, so hinkt es auf dem betreffenden Vorderbein, indem es mit demselben im Ellbogen einknickt. Statt mit den Zehenballen tritt es mit der ganzen Sohle, ja häufig sogar mit dem ganzen Unterarm bis zum Ellbogen auf. Indem hierbei der Vorderkörper des Tieres sich dem Boden nähert, ist der Schritt, den dabei das intakte Vorderbein ausführt, verkürzt, während der Schritt mit dem injizierten Bein, während dessen das Normalbein den Vorderkörper in normalem Abstand vom Grunde stützt, länger ausfällt ${ }^{1}$ ). Infolgedessen findet das Laufen mit den Vorderbeinen im Jambentakt statt. Úberhaupt können die Tiere trotz des Tonusverlustes im einen Triceps sehr schnell laufen. Sie straucheln dabei wohl einmal nach der Seite des injizierten Beines, kommen aber doch

1) Nicht zu verwechseln mit der S. 186 besprochenen Ataxie. 
schnell vorwärts. Die aktive Kontraktionsfähigkeit des betreffenden Triceps ist dabei vollständig erhalten. Davon kann man sich erstens beim Laufen des Tieres überzeugen, wobei der Ellbogen stark gebeugt und gestreckt wird. Je nach dem Temperament der betreffenden Katze kann man die aktive Beweglichkeit auf verschiedene Weise prüfen. Beim Halten in der Luft in Bauch- oder Rückenlage, bei Versuchen des Tieres, zu kratzen, beim Kneifen der Pfote des anderen Beines, bei starker Dorsalbeugung des Kopfes, wenn das Tier auf dem Tische sitzt, und dergleichen kommt es zu kräftiger Streckung des Ellbogens durch aktive Kontraktion des Triceps, wovon man sich durch Betasten desselben und durch den Widerstand beị Beugung des Ellbogens überzeugen kann. Diese Kontraktion ist aber immer nur von kurzer Dauer, solange eben die betreffende kurze Bewegung ausgeführt wird, und macht dann wieder dem früheren Zustand der Tonuslosigkeit Platz.

Der Grad, bis zu welchem im Einzelfalle der Tonus des eingespritzten Triceps schwindet, wechselt in den verschiedenen Versuchen. Meist ist der Tonus vollständig aufgehoben, oder es sind, im Vergleich zur Normalseite, nur noch geringe Spuren vorhanden. In einzelnen Fällen bleibt etwas mehr Tonus zurück, wobei es dann zweifelhaft bleibt, ob die Dosis ungenügend war, oder ob der Triceps in diesem Falle seinen Tonus reflektorisch mehr aus anderen Quellen bezog. Stets aber war in allen Versuchen ein ausserordentlich deutlicher Tonusverlust des injizierten Triceps nachzuweisen.

Die Deutung der Versuche stösst nach den Ergebnịssen der vorhergehenden Abschnitte auf keine Schwierigkeiten. Der Tonus des Skelettmuskels ist nach Brondgeest reflektorisch bedingt. Sherrington vor allem hat betont, dass die hauptsächlichste Tonusquelle für einen einzelnen Muskel in den proprioceptiven Erregungen zu suchen ist, die aus diesem Muskel selbst stammen. Ausserdem kommen aber, genau wie bei der Enthirnungsstarre (siehe oben S. 178), von anderen Körperstellen ausgehende reflektorische Erregungen hinzu. Durch die verwendeten kleinen Novokaindosen werden die sensiblen proprioceptiven Nervenenden im Triceps gelähmt, ohne dass die Funktion der motorischen Nervenenden beeinträchtigt wird. Der Erfolg ist, dass der proprioceptive tonische Reflex rom Triceps auf den Triceps aufgehoben wird, und dass nur noch der Rest von Tonus überbleibt, welcher von anderen sensiblen Nerven ausgelöst wird, und welcher von Fall zu Fall an Stärke wechselt.

Der Schluss, dass der Tonus eines bestimmten Skelettmuskels eine Folge hauptsächlich der sensiblen Erregungen aus diesem Muskel selber sei, beruht bisher auf Versuchen mit Durchschneidung der Hinterwurzeln, wobei es natürlich niemals möglich gewesen ist, die proprio- 
ceptiven Bahnen von einem einzelrien Muskel getrennt zu durchschneiden. Daher sind die hier geschilderten Experimente von besonderem Interesse, weil es in ihnen zum ersten Male gelungen ist, die Proprioceptoren eines einzelnen Muskels bei erhaltener Motilität zu lähmen und die Folgezustände festzustellen. Das Ergebnis ist über Erwarten deutlich ausgefallen und spricht für die Richtigkeit der Anschauung von Sherrington: der Tricepstonus ist hauptsächlich ein proprioceptiver Reflex vom Triceps selbst.

Durchschneidet man die Hinterwurzeln zu einem Vorderbein, so ist der Triceps in den ersten Tagen ganz schlaff bei erhaltener aktiver Beweglichkeit. Nach etwa einer Woche bekommt der Muskel aber wieder Tonus, der im Laufe der Zeit dann weiter zunimmt und durch tonische Reflexe aus anderen Körpergegenden bedingt ist. Bei diesem - und ähnlichen Versuchen erhebt sich die Frage, ob der erste tonuslose Zustand des Muskels ausschliesslich durch die Durchtrennung der sensiblen Nerven oder durch den Schock der Operation bedingt ist, und ob andererseits der nach einiger Zeit wieder auftretende Tonus auf Abklingen des Schocks beruht oder darauf, dass die Zentren des betreffenden Muskels für die anderen afferenten Erregungen im Laufe der Zeit erregbarer werden. Es erhebt sich also hier dieselbe Frage, wie sie von $\operatorname{Munk}^{1}$ ), Trendelenburg ${ }^{2}$ ) u. a. für die Folgen der Querdurchtrennung des Rückenmarkes erörtert worden ist. Der Ausfall der hier geschilderten Versuche spricht sicherlich dafür, dass die proprioceptiven Erregungen vom Muskel selbst in der Norm-den Hauptanteil an dem Entstehen des Muskeltonus besitzen, dass nach ihrem Fortfall der Muskel ganz oder nahezu schlaff wird, und dass, wenn nach einer Hinterwurzeldurchschneidung nach einiger Zeit wieder Muskeltonus auftritt, dieses auf einer zunehmenden Erregbarkeit der betreffenden motorischen Zentren für afferente Erregungen beruht. Denn man kann sich nicht vorstellen, dass nach der peripheren Lähmung der sensiblen Muskelnerven durch Novokain Schock im Zentrum auftritt.

Hierfür spricht auch, dass mit dem Abklingen der Novokainwirkung alsbald die Funktion sich wieder vollständig herstellt. Gewöhnlich dauert der oben geschilderte Symptomenkomplex 15-20 Minuten. Nach $1 / 2-3 / 4$ Stunde sind Tonus und Bewegungen im injizierten Vorderbein wieder völlig normal.

Die bisherige Schilderung gilt im wesentlichen für den Triceps. Dasselbe Symptomenbild lässt sich aber, wenn auch nicht mit der-

1) H. Munk, Über das Verhalten der niederen Teile des Cerebrospinalsystems nach der Ausschaltung höherer Teile. Berl. Akad. Sitzungsber. 1909 (2), 1106.

2) W. Trendelenburg, Die Methode der reizlosen Ausschaltung am Gehirn und die Theorien der physiol. Hirnpathologie. Z. ges. exp. Med. Bd. 3 S. 328. 1914. 
selben Deutlichkeit, am Gastrocnemius hervorrufen, wie ein Versuch zeigte, in welchem 'Tonusverlust des betreffenden Fussgelenkes bei passiver Beugung, Sohlenstand, Einknicken beim Laufen, Hinken und Sohlengang bei erhaltener aktiver Beweglichkeit beobachtet wurde. Doch ist nach den bisher gemachten Erfahrungen für Demonstrationen der Triceps vorzuziehen, bei welchem der geschilderte Erfolg mit grosser Regelmässigkeit eintritt, während am Hinterbein die anatomische Anordnung der Muskeln derartig ist, dass wahrscheinlich Tonusverluste eines einzelnen Muskels weniger schwere Symptome machen.

Nach Durchschneidung der Hinterwurzeln zu einem Vorderbein ist neben der Muskelschlaffheit die hervorstechendste. Folgeerscheinung die Ataxie, welche sich in einem abnorm grossen Ausmaasse der Bewegungen äussert, so dass beim Laufen die Vorderpfote sehr weit nach oben und vorn, manuhmal bis über das Ohr hinaus, gehoben wird. Nach Novokaininjektion in den Triceps tritt eise derartige Ataxie niemals auf. Es war deshalb zu untersuchen, ob, wenn man ausser dem Triceps noch einige andere wichtige Muskeln and Gelenke des Armes asensibel macht, dann Ataxie auftritt. Zu diesem Zwecke wurden in verschiedenen Kombinationen und insgesamt der Triceps, Biceps', Supraspinatus, Infraspinatus, Subscapularis sowie Schulter-, Ellbogen- und Handgelenk mit Novokain eingespritzt. Danach trat völlige Erschlaffung des Ellbogens, Erschlaffung der Schulterstrecker, unvollständige Erschlaffung der Schulterbenger auf. Das betreffende Vorderbein konnte in Schulter und Ellbogen gut aktiv bewegt werden und nahm beim Stehen und Laufen ganz abnorme Stellungen an. Die oben erwähnten ausfahrenden ataktischen Bewegungen waren aber nicht zu beobachten. Ob das Auftreten derselben durch die erhaltene Sensibilität der übrigen, nicht eingespritzten Muskeln (oder der Haut ?) verhindert wurde, ist ohne besondere Versuche nicht zu entscheidea.

Die in diesem Abschnitte beschriebenen Versuche haben gezeigt, dass durch Einspritzung kleiner Novokaindosen (durchschnittlich $1 \mathrm{ccm}$ pro Kilogramm $1 \%$ iger Lösung) in den Triceps von Katzen bei völlig erhaltener aktiver Beweglichkeit Erschlaffung des Muskels: Tonusverlust und dadurch bedingte charakteristische Bewegungsstörungen auftreten, welche auf eine vorübergehende Lähmung der proprioceptiven Muskelnerven zurückgeführt werden müssen.

Da es demnach durch Novokaineinspritzung in den Muskel möglich ist, einen Zustand voräbergehend zustande zu bringen, der bisher beim Menschen nur durch die Förster'sche Operation (Hinterwurzeldurchschneidung) erreicht werden konnte, so erscheint es wünschenswert, dieses Verfahren auch klinisch zu verwenden, sei es zu diagnostischen 
Zwecken, um festzustellen, ob eine vorhandene Muskelspannung durch proprioceptive Reflexe bedingt ist oder auf Kontraktur beruht, sei es bei orthopädischen Maassnahmen, wenn man einen Muskelspasmus vorübergehend aufheben will, was bisher nur durch tiefste Allgemeinnarkose möglich war bzw. die Tenotomie nötig machte. Über die praktischen Ergebnisse dieses Verfahrens beim Menschen kann hoffentlich in einer folgenden Mitteilung berichtet werden ${ }^{1}$ ).

\section{Die Wirkung des Novokains anf den tetanusstarren Skelettmuskel.}

Nachdem sich in den bisher geschilderten Versuchen ergeben hatte, dass kleine Novokaindosen die proprioceptiven Muskelnerven lähmen, und aass es hierdurch sowohl beim normalen wie beim dezerebrierten Tier zu einer hochgradigen Muskelerschlaffung bei völlig unverändert erhaltener Motilität kommt, musste nunmehr untersucht werden, $\mathrm{ob}$ diese selbe Wirkung auch am tetanusstarren Muskel eintritt, und welcher Einfluss dadurch auf die Starre ausgeübt wird.

Im ganzen wurden bei elf Katzen, deren Gewicht von $0,6-3,7 \mathrm{~kg}$ schwankte, Dosen von $0,3-1 \mathrm{ccm}$ einer $1 / 4 \%$ igen Lösung eines gut wirksamen Tetanustrockentoxins in den Muskelbauch des Triceps eingespritzt. Dieses Verfahren hat den Vorteil, dass sich die Starre anfangs ausschliesslich auf den injizierten Triceps" beschränkt und erst sehr spät und in schwäsherem Grade auf benachbarte Muskeln, den Biceps und die SchultermuskeIn, übergreift. Die Starre begann, je nach der Grösse der Tiere und der Höhe der Dosis, nach 2-3Tagen, nur einmal war (bei einem Tiere von 1,3 kg) bereits nach 24 Stunden der erste Anfang der Starre nachzuweisen. Wir haben dann die Tiere in wechselnden Zeiten nach dem Starrebeginn, bis zu 7 Tagen nachher, untersucht. Niemals kam es dabei zu allgemeinem Tetanus, nur in einem Falle befand sich das Tier gerade an der Grenze des Auftretens von allgemein gesteigerter Reflexerregbarkeit.

Die Starre entwickelte sich in der Weise, dass anfangs nur ein gesteigerter Widerstand gegen passive Beugung im Ellbogengelenk nachweisbar war, dass aber der Ellbogen bei Willkürbewegungen des Tieres und nach Kneifen der Pfote noch vollständig gebeugt werden konnte, wobei der vorher deutlich starre Muskel vorübergehend total erschlaffte; daran schloss sich dann ein Stadium, in welchem der Triceps bei derartigen Beugebewegungen nur noch teilweise, aber nicht mehr vollständig erschlaffte, und schliesslich erfolgte (nach 5 bis

1) Inzwischen hat sich herausgestellt, dass man in Fälen von spastischer Lähmung an Menschen durch Einspritzen von 1\% igem Novokain die Spasmen bei erhaltener aktiver Beweglichkeit aufheben oder hochgradig vermindern kann. (Klinik von Prof. Winkler.) 
6 Tagen) weder spontan noch auf Pfotenkneifen eine Beugung des Ellbogens und eine Erschlaffung des Triceps. Daraus folgt, dass im Beginn der Starre der Triceps bei Beugebewegungen des Ellbogens noch durch reciproke Innervation (Sherrington) gehemmt werden kann, dass also die durch Sherrington gefundene Aufhebung der reciproken Hemmung durch Tetanustoxin ${ }^{1}$ ) in den ersten Stadien der Starreentwicklung noch nicht vorhanden ist und sich erst allmählich ausbildet, also ein sekundärer Prozess ist, welcher erst später dazu kommt.

Die Starre war in allen unseren Fällen rein zentral bedingt und war nicht durch die von Gumprecht ${ }^{2}$ ) und von Fröhlich and H. Meyer ${ }^{3}$ ) beschriebene sekundäre Verkürzungskontraktur kompliziert. Sie schwand nach Durchschneidung des Plexus brachialis vollständig (in zwei Fällen nach zweitägiger Dauer der Starre, einmal nach 3 Tagen, zweimal nach 5 Tagen). In einem Versuche liess sie sich durch Einspritzung einer grossen Novokaindosis, welche die indirekte Erregbarkeit des Triceps vom Nerven aus vernichtete, vollständig aufheben.

Nach unseren Erfahrungen können wir daher die Methode der intramuskulären Impfung mit Tetanustoxin für das Studium der lokalen Muskelstarre durchaus empfehlen.

In einer ersten Versuchsreihe wurden die Katzen ein seitig injiziert und darauf in sehr verschiedenen Stadien von eben beginnender bis zu hochgradiger Starre von mehrtägiger Dauer untersucht. Wir haben die Tiere nicht dezerebriert, weil die Enthirnungsstarre das Bild der Tetanusstarre sonst getrübt hätte. Sie wurden vielmehr dekapitiert. Man erhält dann ein sehr gutes Reflexpräparat ohne Enthirnungsstarre, bei welchem der Unterschied zwischen dem tetanusstarren Triceps der einen und dem völlig schlaffen, aber reflektorisch gut erregbaren Triceps der anderen Seite ausserordentlich deutlich ist und sowohl durch den Widerstand gegen passive Beugung im Ellbogen wie durch die verschiedene Haltung der Vorderbeine bei symmetrischer Rückenlage des Tieres mit Schärfe festzustellen ist. Zur Gewinnung eines zahlenmässigen Ausdruckes bestimmten wir auch hier jedesmal den Winkel, bei welchem bei passiver Beugung im Ellbogen zuerst ein' muskulärer Widerstand im Triceps fühlbar wurde. Dann wurde in den tetanusstarren Triceps $1 \%$ ige Novokainlösung eingespritzt und,

1) C. S. Sherrington, On reciprocal innervation of antagonistic muscles. 8. note. Proc. Roy. Soc. B. vol. 76 p. 269.1905.

2) F. Gumprecht, Versuche über die physiol. Wirkungen des Tetanusgiftes im Organismus. Pflüger's Arch. Bd. 59 S. 105. 1894.

3) A. Fröhlich u. H. H. Meyer, Untersuchungen über den Tetanus. Schmiedeberg's Arch. Bd. 79 S. 55. 1915. 
festgestellt, bei welchen Novokaindosen eine teilweise und eine vollständige Lösung der Starre erfolgte. Meist wurde zur Kontrolle auf der normalen Seite die gleiche Menge 0,9\% ige Kochsalzlösung injiziert, die stets ohne jede Wirkung blieb.

Zur Prüfung der reflektorischen Erregbarkeit des Triceps benutzten wir entweder den gekreuzten Streckreflex auf Kneifen der Pfote des gegenseitigen Vorderbeines, meist aber den hierfür sehr geeigneten, auf S. 173 beschriebenen ,Tricepsreflex" auf Beklopfen des Endgliedes des zu prüfenden Vorderbeines, worauf eine Streckung des Ellbogens erfolgt, die durch Tricepskontraktion bedingt ist, welche sich meist durch Betasten des Triceps durch die Haut hindurch als solche direkt fühlen lässt.

Nach vollständiger Lösung der Starre wurde beiderseits der Plexus brachialis freigelegt, der Triceps von Haut entblösst und der Schwellenwert für die indirekte faradische Erregbarkeit des Triceps und für die direkte Erregbarkeit in Kronecker - Einheiten festgestellt.

Die folgenden Protokolle mögen als Beispiele für das Verhalten bei beginnender und bei sehr hochgradiger Tetanusstarre dienen:

$\nabla$ ersuch IX. Kätzchen von $1 \mathrm{~kg}$.

18. Nov. $11^{\mathrm{h}}$ vorm. Injektion von $0,35 \mathrm{cem}{ }^{1 / 4} \%$ iger Tetanustoxinlösung in den rechten Triceps.

19. "Noch keine Starre mit Sicherheit nachzuweisen.

20. \# $\quad 9^{1 / 2} \mathrm{~h}$. Deutliche Starre im rechten Triceps. Widerstand im Ellbogen bei etwa $90^{\circ}$. Streckstand des rechten Vorderbeines, wenn das Tier frei in der Luft gehalten wird. Das Bein kann aber aktiv ziemlich vollständig gebeugt werden. Das Tier läuft gut.

20. $" 10^{\mathrm{h}}$. Äthernarkose, Tracheotomie, Vagi durchtrennt, Karotiden abgebunden. Dekapitieren nach Sherrington. Danach Äther abgestellt. Tonische Starre im rechten Ellbogen deutlich. Widerstand bei etwa $70-45^{\circ}$.

20. " $11^{\mathrm{h} 15}$. Lebhafte Reflexe des Hintertieres (Patellarreflexe, Kratzreflex, Laufbewegungen). Linkes Vorderbein in Rückenlage vollständig gebeugt, ohne Tonus. Am rechten Vorderbein steht der Untersirm senkrecht nach oben, Ellbogen $135^{\circ}$, deutliche Starre des Triceps. An beiden Vorderbeinen lebhafte gleichseitige Beugreflexe.

20. " 11 h $30,{ }^{1 / 2} \mathrm{ccm} \mathrm{1 \%} \%$ iges Novokain in den rechten Triceps, $1 / 2 \mathrm{ccm}$ phys. NaCl-Lösung in den linken Triceps injiziert.

20. " $11^{\mathrm{h}} 35$ '. Stellungsunterschied der beiden Vorderbeine noch deutlich. Widerstand gegen passive Beugung im rechten Ellbogen etwas vermindert.

20. $" 11^{\mathrm{h}} 37^{\prime}$. $1 / 2 \mathrm{ccm} 1 \%$ iges Novokain in den rechten, $1 / 2 \mathrm{ccm} \mathrm{NaCl}$ in den linken Triceps.

20 . " $11^{\mathrm{h}} \mathrm{42}$ '. Stellungsunterschied bëider Vorderbeine verschwunden. Bei passiver Beugung noch ein etwas grösserer Widerstand im rechten Ellbogen als im linken.

20. $11^{\mathrm{h}} 45^{\prime} \cdot 1 / 2 \mathrm{ccm} \mathrm{1 \%}$ iges Novokain in den rechten, $1 / 2 \mathrm{ccm} \mathrm{NaCl}$ in den linken Triceps.

20. " $11^{\mathrm{h}} 50^{\prime}$. In Ruhe kein Stellungsunterschied und kein Widerstandsunterschied im rechten und linken Ellbogen. Auf Kneifen beider 
Vorderpfoten rechter Ellbogen etwas mehr gestreckt als links, aber kein stärkerer Widerstand rechts als links. Tricepsreflex beiderseits deutlich. Nach einer Minute Stellungsunterschied verschwunden.

20. Nov. $11^{\mathrm{h}} 55^{\prime}$. 1/4 $\mathrm{ccm} 1 \%$ iges Novokain rechts, $1 / 4 \mathrm{ccm} \mathrm{NaCl}$ links in den Triceps.

20. "12h. Stellungsunterschied völlig geschwunden. Kein Widerstandsunterschied gegen passives Beugen beider Ellbogen, auch nicht nach Pfotenkneifen. Tricepsreflex beiderseits deutlich.

Präparation des Plexus brachialis und Freilegung. des Triceps beiderseits. Faradische Reizung, zwei Akkumulatoren, kein Extrawiderstand im sek. Kreis.

Erregbarkeit: indirekt links $400 \mathrm{Kr}$, rechts $600 \mathrm{Kr}$, " direkt " $800 "$ " $1750 "$

Danach Tricepsreflex beiderseits noch deutlich vorhanden, verschwindet auf Durchtrennung des Plexus.

Ergebnis: Die 2 Tage nach intramuskulärer Toxineinspritzung deutlich ausgesprochene lokale Starre des Triceps ist nach dem Dekapitieren unverändert erhalten. Sie nimmt nach Einspritzung von $1 / 2 \mathrm{ccm} 1 \%$ igem Novokain wenig, nach $1 \mathrm{ccm}$ fast völlig ab und ist nach $13 / 4$ ccm total aufgehoben. Dabei lässt sich aber der Muskel im Tricepsreflex noch unverändərt gut rom Zentrum aus erregen, die indirekte und direkte faradische Erregbarkeit sind noch gut erhalten (wenig herabgesetzt).

Versuch X. Katze $1,9 \mathrm{~kg}$.

18. Nov. $11^{\text {h }}$ vorm. $0,35 \mathrm{ccm} 1 / 4 \%$ iges Tetanustoxin in den rechten Triceps.

19. ". Keine Starre.

20. " Starre im rechten Triceps wird deutlich.

21. " Starre stärker.

22. " Sehr starke Starre im rechten Triceps. Starker Widerstand bei $90^{\circ}$. Kann noch gut springen und klettern, setzt aber die rechte Vorderpfote manchmal mit dem Rücken auf.

23. " Sehr kräftige Starre des rechten Ellbogens, nicht im Fussgelenk, dagegen wahrscheinlich etwas in der Schulter, nicht im Biceps. Läuft und klettert unter Mitbenutzung des rechten Vorderbeins, welches dabei noch bis auf $90^{\circ}$ gebeugt werden kann. Bei Kopfsenken in Fussstellung kann der rechte Ellbogen selbst bis auf $70^{\circ}$ gebeugt werden. Sonst ist der Widerstand des Ellbogens aber bei $135^{\circ}$. Starre ausserordentlich kräftig. Kein allgemeiner Tetanus, keine Steigerung der Reflexerregbarkeit.

23. " $10^{\mathrm{h}}$. Dekapitieren in Äthernarkose, die danach abgestellt wird. Sofort danach beiderseits gute Tricepsreflexe. Widerstand im rechten Fillbogen bei $80^{\circ}$.

23. " $10 \mathrm{~h} 31^{\prime} .1 \mathrm{ccm} 1 \%$ iges Novokain rechts, $1 \mathrm{ccm} R$ inger links in den Triceps ${ }^{1}$ ).

23. " $10^{\mathrm{h}} 36^{\prime}$. Starre nur wenig vermindert, Widerstand bei $50^{\circ}$. Tricepsreflex beiderseits gut.

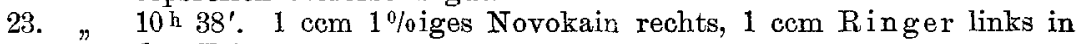
den Triceps.

1) Die Dosis wurde doppelt so gross genommen als im vorigen Versuch, weil das Tier etwa doppelt so schwer war. 
23. Nov. 10 $44^{\prime}$. Starre hat deutlich abgenommen, ist aber nicht völlig geschwunden. Widerstand bei $40-45^{\circ}$. Tricepsreflex deutlich. Die Starre ist zum grösseren Teil geschwunden, aber ein zweifelloser Rest ist noch vorhanden.

23. $" 10^{\mathrm{h}} 46^{\prime} .1 \mathrm{ccm} \mathrm{1 \%}$ iges Novokain rechts, $1 \mathrm{ccm} R$ inger links in den Triceps.

23. " $10^{\text {h }} 53^{\prime}$. Starre gering, aberdoch noch etwas vorhanden. Geringer Widerstand bei etwa $30^{\circ}$. Tricepsreflexrechts sehr deutlich, stärker als links. In Rückenlage ist der rechte Ellbogen nur wenig mehrgestrecktals derlinke.

23. " $10^{\mathrm{h}} 58^{\prime} .1 \mathrm{ccm} 1 \%$ iges Novokain rechts, $1 \mathrm{ccm}$ Ringer links in den Triceps.

23. " $11^{\mathbf{b}} 8^{\prime}$. Starre rechts vollständig geschwunden. Kein Stellungsunterschied in Rückenlage, kein Widerstandsunterschied im rechten und linken Ellbogen. Tricepsreflex links deutlich; rechts erfolgt dagegen keine Bewegung im Triceps, nur in der Schulter.

Beiderseits Freilegung des Plexus brachialis und des Triceps.

Faradische Erregbarkeit: indirekt links 100-150 Kr., rechts unerregbar $(2000 \mathrm{Kr}$. unsichere Reaktion),

$" \quad$ direkt links $900 \mathrm{Kr}$., rechts $1500 \mathrm{Kr}$.

Ergebnis: Die Starre des Triceps beginnt 2 Tage nach der Toxininjektion und ist nach 5 Tagen sehr stark ausgebildet. Sie bleibt nach dem Dekapitieren erhalten. Nach $1 \mathrm{ccm} 1 \%$ igem Novokain wird sie wenig, nach 2 und $3 \mathrm{ccm}$ dagegen sehr beträchtlich und bis auf einen kleinen Rest vermindert. Dabei ist die reflektorische Erregbarkeit des Triceps vom Zentrum aus noch unvermindert erhalten. Erst auf $4 \mathrm{ccm}$ schwindet die Starre vollständig, die reflektorische Erregbarkeit und die indirekte faradische Erregbarkeit vom Nerven aus sind dann erloschen, die direkte Muskelerregbarkeit dagegen erhalten.

Die übrigen Versuche führten zu dem gleichen Ergebnis. Sie sind hier in Tabellenform zusammengestellt.

\begin{tabular}{|c|c|c|c|c|c|c|c|c|}
\hline \multirow{2}{*}{$\mathrm{Nr}$. } & \multirow{2}{*}{$\begin{array}{c}\text { Ge- } \\
\text { wicht } \\
\text { kg }\end{array}$} & \multirow{2}{*}{$\begin{array}{l}\text { Novo- } \\
\text { kain- } \\
\text { dosis } \\
\text { ccm. }\end{array}$} & \multirow{2}{*}{$\begin{array}{c}\text { Dosis } \\
\text { pro } \\
\mathrm{kg} \\
\mathrm{ccm}\end{array}$} & \multicolumn{2}{|c|}{$\begin{array}{l}\text { Indirekte Er- } \\
\text { regbarkeit }\end{array}$} & \multicolumn{2}{|c|}{$\begin{array}{c}\text { Direkte Erreg- } \\
\text { barkeit }\end{array}$} & \multirow{2}{*}{$\begin{array}{c}\text { Starre } \\
\text { schwindet }\end{array}$} \\
\hline & & & & $\begin{array}{l}\text { Novo- } \\
\text { kain- } \\
\text { seite }\end{array}$ & $\begin{array}{l}\text { Nor- } \\
\text { mal- } \\
\text { seite }\end{array}$ & $\begin{array}{l}\text { Novo- } \\
\text { kain- } \\
\text { seite }\end{array}$ & $\begin{array}{l}\text { Nor- } \\
\text { mal- } \\
\text { seite }\end{array}$ & \\
\hline 4 & 0,60 & $1^{3 / 4}$ & 2,9 & 500 & 250 & 2000 & & \\
\hline 7 & 1,25 & $1^{1 / 2}$ & 1,2 & 70 & 50 & 2250 & 1750 & total \\
\hline 24 & 3,6 & 3 & 0,8 & 15 & 25 & 1000 & 1000 & fast völligg \\
\hline 29 & 1,8 & 1 & 0,8 & 50 & 50 & 400 & 500 & total \\
\hline 30 & & 4 & 1 & 20 & 20 & 400 & 400 & \\
\hline 31 & 2,7 & 5 & 1,85 & 10 & 20 & 200 & 300 & $"$ \\
\hline
\end{tabular}

Die Versuche 4 und 7 dieser Tabelle sind genau in der gleichen Weise angestellt wie die oben ausführlich als Protokolle wiedergegebenen Versuche 9 und 10. In den übrigen Versuchen 24-31 war das Tetanus- 
toxin beiderseits in den Triceps injiziert; nach Entwicklung der Starre wurde dekapitiert und darauf Novokain nur an einer Seite eingespritzt (an der anderen Seite wurden die Hinterwurzeln durchschnitten), so dass am Schlusse die faradische Erregbarkeit mit und ohne Novokain an zwei Muskeln verglichen werden konnte, welche beide unter dem Einflusse von Tetanustoxin gestanden hatten. Gerade in diesen letzteren vier Versuchen ist nun durch Novokaindosen, welche die Starre dreimal total und einmal fast völlig zum Verschwinden brachten, wobei stets die Erregbarkeit des Muskels vom Zentrum aus unbeeinträchtigt blieb, die indirekte faradische Erregbarkeit des Triceps vom Nerven aus quantitativ unvermindert geblieben. Diese Versuche sind also gerade besonders beweisend. In den Versuchen 4-10, in welchen das Toxin nur einseitig injiziert worden war, fand sich auf der Seite der Tetanusstarre nach Injektion kleiner Novokaindosen meist eine etwas, wenn auch nur unbedeutend geringere indirekte und direkte Erregbarkeit als auf der Normalseite. Hierdurch wird es wahrscheinlich, dass diese leichte Verminderung der Erregbarkeit nicht auf Kosten des Novokains, sondern des Tetanustoxins zu setzen ist, besonders da durch Gumprecht gezeigt wurde, dass bei längerer Dauer der Tetanusstarre die Erregbarkeit des Muskels durch den elektrischen Strom allmählich abnimmt.

Diese Versuche zeigen also übereinstimmend, dass es durch die verwendeten kleinen Novokaindosen geradeso wie bei der Enthirnungsstarre so auch bei der Tetanusstarre gelingt, die Dauerkontraktion des Muskels entweder total oder in anderen Versuchen bis auf einen kleinen unbedeutenden Rest zum Verschwinden zu bringen, ohne die reflektorische Erregbarkeit vom Zentrum aus zu beeinträchtigen, und ohne die indirekte faradische Erregbarkeit vom Nerven aus wesentlich zu vermindern. Letztere kann vielmehr quantitativ unverändert bleiben.

Damit ist der tatsächliche Befund, den E. Meyer und Weiler am Biceps des Menschen erhoben haben, vollinhaltlich beim Tiere bestätigt worden.

Nachdem sich herausgestellt hatte, dass man die lokale tetanische Muskelstarre beim dekapitierten Tiere durch kleine Novokaindosen lösen kann, welche den Muske! und den motorischen Nerven nicht lähmen, musste nunmehr geprüuft werden, ob dasselbe auch beim intakten Tiere mit erhaltenem Gehim gelingt. Es ergab sich, dass dieses tatsächlich der Fall ist.

Zwei Versuchsprotokolle mögen als Beispiel dienen. 
Versuch XXIX. Katze $1,28 \mathrm{~kg}$.

14. Jan. $19195^{1 / 2}$ h. Einspritzung von je $0,4 \mathrm{ccm} 1 / 4 \%$ iger Tetanustoxinlösung beiderseits in den Triceps.

16. "Beginnende Starre des rechten Triceps, links noch keine Starre.

17. " Beiderseits' Starre im Triceps. Bei Rückenlage des Tieres Ellbogen links $90^{\circ}$, rechts $170^{\circ}$.

17. $" 4^{\mathrm{h}} 57^{\prime} .1 \mathrm{ccm} 1 \%$ iges Novokain in den rechten Triceps.

17. " $" 5 \mathrm{~h}$. In Rückenlage linker Ellbogen $90^{\circ}$, rechter Ellbogen $45^{\circ}$ (?). Starkes Einknicken beim Laufen im rechten Ellbogen. Kurz darauf Starre vollständig geschwunden. Sohlengang rechts. Bei Druck auf das linke Schulterblatt beim stehenden Tiere fühlt man einen kräftigen Widerstand, bei Druck auf das rechte Schulterblatt dagegen keinen Widerstand, und das Tier knickt ein. Wird das Tier an Kopf und Becken gehalten und mit einem Vorderbein auf den Tisch gestellt, so kann es auf dem linken Vorderbein stehen, auf dem rechten dagegen nicht und knickt sofort ein. Sehr starkes Hinken mit dem rechten Vorderbein beim Laufen. - Der rechte Ellbogen kann aber aktiv völlig gestreckt werden.

17. $" 5^{\mathrm{h}} 7^{\prime}$. Läuft schnell durch das Zimmer unter starkem Hinken mit dem rechten Vorderbein. Rechøer Triceps völlig schlaff. B Beim Kneifen der linken Pfote wird der rechte Ellbogen völlig gestreckt.

17. . "5 h $15^{\prime}$. Tonus im rechten Triceps kehrt allmählich zurück. Hinkt nicht mehr. Kein Sohlengang. In Rückenlagge beide Ellbogen $90^{\circ}$.

17. $" 5^{\text {h }} 30^{\prime}$. Starre im rechten Ellbogen rechts wieder stärker als links. $\nabla$ ersuch XXX. Katze $3,4 \mathrm{~kg}$.

20. Jan. $19192^{1 / 2} \mathrm{~h}$. Injektion von je $1 \mathrm{ccm} 1 / 4 \%$ igem Tetanustoxin beiderseits in den Triceps.

23. " Beginnende Starre im rechten Triceps.

24. "Beiderseits Starre im Triceps.

27. " Sehr deutliche Starre beiderseits (ca. 110-135\%).

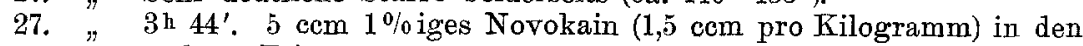
rechten Triceps.

27. " $3 \mathrm{~h} 46^{\prime}$. Einknicken mit dem rechten $\nabla$ orderbein boim Laufen. Rechter Ellbogen passiv völlig beugbar, was vorher nicht möglich war. Sohlengang des rechten Vorderbeines.

27. $" 3^{\mathrm{h}} 50^{\prime}$ Tonus des rechten Triceps völlig geschwunden. Kann auf dem rechten Vorderbeine nicht mehr stehen und knickt sofort ein. Stärkstes Hinken. Tritt beim Laufen mit dem rechten Vorderbein bis zum Ellbogen auf. Aktive Streckung des rechten Ellbogens deutlich vorhanden.

27. " $4^{\text {h }} 10^{\prime}$. Starre des rechten Triceps beginnt zurückzukehren.

27. " $" 5^{\text {h }} 45^{\prime}$. Rechter Ellbogen $135^{\circ}$, starke Starre des rechten Triceps.

Diese beiden Versuche zeigen, dass Einspritzen von kleinen Novokaindosen $(0,8-1,5 \mathrm{cem} 1 \%$ ige Lösung pro Kilogramm) in den tetanusstarren Triceps diesen in genau derselben Weise zur Erschlaffung bringt, wie das beim Triceps normaler Katzen der Fall ist und oben S. 183 eingehend geschildert wurde. Auch die Bewegungsstörungen sind genau die gleichen wie bei normalen Tieren. 
Das Ergebnis sämtlicher derartiger Versuche ist in nachstehender Tabelle zusammengefasst.

\begin{tabular}{|c|c|c|c|c|c|}
\hline \multirow[t]{2}{*}{$\mathrm{Nr}$. } & \multirow{2}{*}{$\begin{array}{c}\text { Dauer der } \\
\text { Starre } \\
\text { Tage }\end{array}$} & \multirow{2}{*}{$\begin{array}{c}\text { Gewicht } \\
\text { der Tiere } \\
\text { kg } \\
\end{array}$} & \multicolumn{2}{|c|}{$\begin{array}{l}\text { Novokaindosis } \\
\text { ccm }\end{array}$} & \multirow{2}{*}{$\begin{array}{l}\text { Erschlaffung } \\
\text { des Triceps }\end{array}$} \\
\hline & & & total & pro kg & \\
\hline $\begin{array}{l}24 \\
25 \\
- \\
-29 \\
30 \\
31\end{array}$ & $\begin{array}{l}2 \\
2 \\
3 \\
2 \\
5 \\
1\end{array}$ & $\begin{array}{l}3,6 \\
3,7 \\
\overline{-} \\
\overline{1,3} \\
3,4 \\
2,7\end{array}$ & $\begin{array}{l}2 \\
2 \\
3 \\
3 \\
1 \\
5 \\
4\end{array}$ & $\begin{array}{l}0,55 \\
0,54 \\
0,8 \\
0,8 \\
0,8 \\
1,5 \\
1,5\end{array}$ & $\begin{array}{l}\text { völlig } \\
\text { etwas } \\
\text { deutlich } \\
\text { völlig } \\
\quad " \\
\text { " }\end{array}$ \\
\hline
\end{tabular}

Man sieht, dass bei vier Tieren die verwendeten kleinen Novokaindosen zu völliger Erschlaffung des in Tetanusstarre befindlichen Triceps führten, während bei einem Tiere die Erschlaffung deutlich, aber nicht total war. Auch hierin verhält sich der also tetanisch starre Muskel wie der normale Muskel.

Da nun in den vorhergehenden Abschnitten gezeigt werden konnte, dass die verwendeten kleinen Novokaindosen den Muskel und den motorischen Nerven intakt lassen, dagegen die proprioceptiven sensiblen Nervenenden im Muskel lähmen, und dass hierauf die am normalen Skelettmuskel beobachteten Novokainwirkungen beruhen, liegt die Schlussfolgerung nahe, dass auch die Lösung der lokalen Muskelstarre beim Tetanus durch Novokain auf der peripheren Lähmung sensibler Muskelnerven beruht.

Wenn dieser Schluss richtig ist, dann würde sich daraus weiter ergeben, dass die lokale Muskelstarre beim Tetanus ausgelöst und unterhalten wird durch sensible Erregungen, welche grösstenteils in den starren Muskeln selbst ihren Ursprung nehmen, und welche nur deshalb zu der abnorm starken Muskelstarre führen, weil durch das Tetanusgift das Zentrum in einen Zustand von Übererregbarkeit versetzt worden ist. (Denn die Annahme, dass das Tetanusgift die, sensiblen Nervenenden im Muskel direkt erregt, darf als widerlegt angesehen werden ${ }^{1}$ ).)

Um diese Folgerung auf ihre Richtigkeit zu prüfen, war es nötig, den Einfluss der Durchschneidung der Hinterwurzeln auf die lokale Tetanusstarre zu untersuchen und am selben Tier den Erfolg der Hinterwurzeldurchschneidung "mit dem der Novokaineinspritzung zu vergleichen.

1) C. Brunner, Deutsche med. Wochenschr. 1894, S. 100. - F. Gum precht, Deutsche med. Wochenschr. 1894, S. 546 und Pflü ger's Arch. Bd. 59 S. 105. 1894. - H. Meyer und F. Ransom, Schmiedeberg's Arch. Bd. 49 S. 369.1903. 
Um einen guten Vergleich zu ermöglichen, wurde zunächst bei vier Katzen beiderseits Toxin in den Triceps eingespritzt und nach Entwicklung einer guten Tetanusstarre an beiden Vorderbeinen die zugehörigen Hinterwurzeln auf einer Seite durchschnitten. Darauf wurden die Tiere dekapitiert und in der üblichen Weise untersucht. Als Beispiel diene folgendes Protokoll:

Versuch XXX. Katze $3,4 \mathrm{~kg}$.

20. Jan. $19192^{1 / 2}$ h. Injektion von je $1 \mathrm{ccm}{ }^{1 / 4} \%$ igem Tetanustoxin in den rechten und linken Triceps.

23. " Beginnende Starre im rechten Triceps.

24. " Starre beiderseits im Triceps.

25. " Deutliche, aber noch nicht maximale Starre der beiden Triceps. Beide Ellbogen können auf Pfotenkneifen noch völlig gebeugt werden. Ellbogen rechts $110^{\circ}$, links $70^{\circ}$.

27. - Sehr deutliche Starre beiderseits $\left(110-135^{\circ}\right)$. Linkes Vorderbein kann nicht mehr ganz gebeugt werden (bis etwa $70^{\circ}$ ). Rechtes Vorderbein kann noch nahezu gebeugt werden. Prüfung der Novokainwirkung am intakten Tier siehe oben S. 193.

28. „ Starre beiderseits sehr kräftig, links $135^{\circ}$, rechts $90^{\circ}$. Links ist aktive Beugung nur bis $70-90^{\circ}$, rechts bis $30^{\circ}$ möglich. Beide Ellbogen können aktiv völlig gestreckt werden. Noch keine allgemein gesteigerte Reflexerrogbarkeit.

28., $9 \mathrm{~h} 30^{\prime}$. Äthernarkose. Karotiden abgebunden, Vagi durchtrennt. Auf der linken Seite werden die Hinterwurzeln von $\mathrm{C}_{5}$ bis Th. unter Schonung der Vorderwurzeln durchtrentt und die Wunde geschlossen. Sofort danach in mitteltiefer Äthernarkose starke Starreim rechten Triceps, während der linke Triceps völlig schlaff ist. Darauf Dekapitieren. Äthernarkose abgestellt. Danach lebhafte Reflexe des Hintertieres. Gute Starre im rechten Triceps $\left(90^{\circ}\right)$, während die anderen Muskeln des rechten Vorderbeines tonuslos sind. Linker Triceps ganz schlaff.

28. $\Rightarrow \quad 10^{\mathrm{h}} 15^{\prime}$. Ende der Operation.

28. " $" 10^{h} 35$ '. Gute Starre des rechten Triceps, linkes Vorderbein ganz schlaff. Lebhafte Reflexe des Hintertieres.

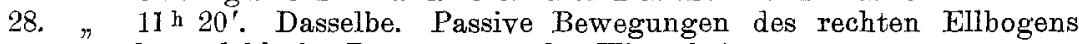
lösen lebhafte Bewegungen der Hinterbeine aus.

28. " $11^{\mathrm{h}} 30^{\prime} .4 \mathrm{~cm} \mathrm{1 \%}$ iges Novokain in den rechten Triceps $(1,2 \mathrm{ccm}$ pro Kilogramm).

28. " $11^{\text {h }} 35$ '. Starre des rechten Triceps fa st völlig geschwunden.

28. , $11^{\mathrm{h}} 37^{\prime}$. Starre des rechten Triceps völlig geschwunden. Wird das Tier am Hinterkörper kräftig angepackt und hin und her bewegt, so kehrt der Tonus des rechten Triceps vorübergehend etwas zurück. Passive Bewegungen des rechten Ellbogens lösen jetzt keine Reflexe auf das Hintertier mehr ans.

Präparation beiderseits des Plexus brachialis und Freilegung des Triceps. Indulstorium mit einem Akliumulator.

Faradische Erregbarkeit des Triceps:

$$
\text { Indirekt: links } 20 \mathrm{Kr} \text {., rechts } 20 \mathrm{Kr} \text {., }
$$

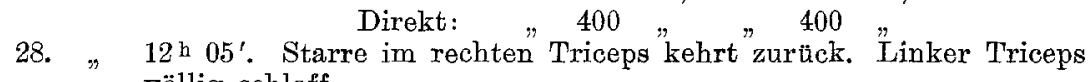
völlig schlaff.

28. " $2^{\mathrm{h}} 07^{\prime}$. Sehr schöne Starre im rechten Ellbogen, über $90^{\circ}$. Bei passiven Bewegungen desselben lebhafte Bewegungen der Hinter- 
beine. Linker Triceps völlig schlaff. Das Tier ist gegen Ende des Versuches an der Grenze der allgemeinen Reflexsteigerung.

28. Jan. $2 \mathrm{~h} 40^{\prime}$. Nach Durchschneidung des rechten Plexus brachialis rechtes Vorderbein völlig schlaff.

28. $" 2$ h $45^{\prime}$. Trachea abgeklemmt. Bei den Erstickungskrämpfen erfolgt maximale Streckung des linken Ellbogens durch Kontraktion des desensibilisierten linken Triceps.

Sektion: Links Hinterwurzeln von $\mathrm{C}_{5}$ bis $\mathrm{Th}_{2}$ völlig durchtrennt, alle Vorderwurzeln intakt.

Die übrigen drei Versuche hatten genau das gleiche Ergebnis. Sie wurden 1, 2 und 4 Tage nach dem ersten Auftreten der lokalen Muskelstarre angestellt.

Hieraus ergibt sich also, dass Durchschneidung der Hinterwurzeln vach dem Eintritt der Muskelstarre dieselbe aufhebt, und dass der Muskel dann auch nach dem Dekapitieren schlaff bleibt. Injiziert man dann in den tetanusstarren Muskel der Normaiseite eine kleine Novokaindosis, so erschlafft auch dieser Muskel. Diese Erschlaffung war in drei unserer Versuche (Nr. 29, 30 und 31) total, so dass der Triceps an beiden Seiten gleich schlaff war. In einem Versuche (Nr. 24) blieb nach Novokain noch eine Spur von Tonus zurück, so dass der Triceps auf der Seite der Hinterwurzeldurchschneidung schlaffer war als auf der Novokainseite.

Diese Versuche zeigen, dass die lokale Muskelstarre nach Tetanusvergiftung sowohl durch kleine Novokaindosen wie durch Hinterwurzeldurchschneidung aufgehoben wird, und stützen die Schlussfolgerung, dass die Wirkung kleiner Novokaindosen bei der Tetanusstarre auf der Lähmung der sensiblen Muskelnerven und dem dadurch be dingten Fortfall proprioceptiver Erregungen beruht, welche die lokale Muskelstarre reflektorisch unterhalten.

Gegen die Versuche mit Hinterwurzeldurchschneidung bleibt der Einwand übrig, dass durch diese Operation ein so grosser Schock gesetzt werde, dass hierdurch die Erschlaffung des tetanischen Muskels vorgetäuscht wird. Dieser Einwand war zu der Zeit, als Brunner und Gumprecht ihre grundlegenden Versuche ausführten, berechtigt, hat aber bei der jetzigen Art des Vorgehens einen grossen Teil seiner Bedeutung vertoren. Jeder, der viel an dekapitierten Tieren experimentiert, weiss, wie auffallend gering an derartigen Prëparaten die Schockerscheinungen selbst nach den schwersten Eingriffen am Zentralnervensystem sind. Hochgradiger Schock wird schon dadurch ausgeschlossen, dass die lokale Starre im Vorderbein der anderen Körperseite so kräftig nach der Wurzeldurchschneidung bleibt, und dass auch die Reflexe hier nicht abgeschwächt sind. Wenn aber das Tetanustoxin die lokale Muskelstartare durch alleinige direkte Einwirkung auf das Rückenmark hervorriefe, ohne dass sensible Erregungen von der 
Peripherie dazukämen, und wenn die Tricepserschlaffung nach Hinterwurzeldurchschneidung allein durch den Schock der Operation hervorgerufen würde, so müsste es bei derartigen Tieren unmöglich sein, vom Zentrum aus durch direkte zentrale Erregungsmittel tonische Muskelkontraktionen hervorzurufen. Das ist nun aber wohl möglich. Bei der Erstickung erfolgt jedesmal kräftige tonische Kontraktion des desensibilisierten Triceps, deren Angriffspunkt bekanntlich im Rückenmark liegt (Versuch Nr. 24, 30 und 31). Ausserdem liessen sich in Versuch Nr. 24 lebhafte reflektorische Kontraktionen des betreffenden Triceps von anderen Körperstellen aus auslösen. Der Einwand, dass die Resultate der Hinterwurzeldurchschneidung durch Schock wesentlich getrübt seien, kann daher vernachlässigt werden.

Um aber ganz sicher zu gehen, wurde in einem weiteren Versuche zuerst die einseitige Hinterwurzeldurchschneidung und erst 10 Tage später die intramuskuläre Tetanustoxin-Impfung vorgenommen. Im Gegensatz zu früheren Experimenten wurde das Gift an beiden Vorderbeinen injiziert, weil man nur dann Gelegenheit hat, am gleichen Tier die Tetanusstarre des normalen und desensibilisierten Muskels zu vergleichen. Den Erfolg ersieht man aus nachstehendem Protokoll.

Versuch XXXIII. Kätzchen $0,9 \mathrm{~kg}$.

21. Jan. 1919. 1/4 mg Atropin. Äther-Chloroformnarkose. An der rechten Seite werden die Hinterwurzeln von $\mathrm{C}_{7}, \mathrm{C}_{8}, \mathrm{Th}_{1}$ und $\mathrm{Th}_{2}$ durchschnitten, Vorderwurzeln intakt.

22. "Rechtes Vorderbein schlaff, aktiv bẹweglich. Ausgesprochene Ataxie mit weitem Ausfahren nach vorn beim Laufen. Radiale Zehe sensibel, entsprechend der nicht durchschnittenen Hinterwurzel $\mathrm{C}_{6}{ }^{1}$ ).

23. " Rechtes Vorderbein noch schlaff, bekommt aber doch gelegentlich schon wieder etwas Tonus in Schulter und Ellbogen. Aktive Beweglichkeit sèhr gut. Sehr starke Ataxie.

27. "Kann auf dem rechten Vorderbein allein etwas stehen, knickt aber deutlich ein. Strecktonus im rechten Ellbogen sehr gering, aber doch manchmal deutlich. Aktive Beugung und Streckung des rechten Ellbogens sehr deutlich. Läuft auf vier Pfoten. Starke Ataxie.

28. " Tonus im rechten Vorderbein sehr wechselnd, manchmal in Schulter und Ellbogen deutlich vorhanden, manchmal vollständig fehlend.

31. " Klettert unter starken Mitbewegungen des rechten Vorderbeines bis auf die Schulter. Rechtes Vorderbein noch immer sohlaff, wird aber manchmal sehr deutlich tonisch innerviert. Besonders lässt sich dieses durch bestimmte Kopfstellungen hervorrufen.

1. Febr. $0,3 \mathrm{ccm} 1 / 4 \%$ iges Tetanustoxin in den rechten und den linken Triceps.

3. "Im linken Triceps deutlich beginnende Starre, Ellbogen kann noch aktiv gebeugt werden. - Der rechte Ellbogen ist schlaffer

1) C. S. Sherringt on, Transact. Roy. Soc. B. vol. 184 p. 685 . Lon: don 1893. 
als der linke, manchmal ganz schlaff, manchmal hat er deutlichen Tonus, wie auch vor der Toxineinspritzung.

5. Febr. Sehr gute aktive Beweglichkeit des rechten Vorderbeines, Sohlengang. Starre im linken Ellbogen sehr deutlich. - Auf dem rechten Vorderbein kann die Katze nicht stehen, sondern knickt sofort im Ellbogen ein. Der rechte Triceps ist viel schlaffer als der linke, hat aber manchmal, nicht immer, deutlichen Tonus. Wenn aber das rechte Vorderbein schlaff ist, ist keine Spur von Starre im rechten Ellbogen fühlbar. Zustand also wie vor der Toxininjektion. Aktive Beweglichkeit des rechten Ellbogens vortrefflich.

6. ” Deutliche Starre des linken Triceps. Starre $90^{\circ}$. - Sehr schöne aktive Beweglichkeit des rechten Vorderbeines mit starker Ataxie. Im rechten Vorderbein sicher keine Starre. Tier kann nicht auf dem rechten Vorderbein allein stehen. Rechter Ellbogen bekommt bei willkürlicher Streckung schönen Tonus.

6. "10h $18^{\prime}$. Injektion von $1 \mathrm{ccm} 1 \%$ igem Novokain in den rechten Triceps.

6. " $10 \mathrm{~h} 25^{\prime}$. Zustand ganz ungeändert. Rechter Ellbogen aktiv beweglich, gut streckbar, bekommt dabei deutlichen Tonus, sonst Triceps aber ganz schlaff. Tier kann nicht auf dem rechten Vorderbein stehen. Klettert gut auf die Schulter.

6. "10 $30^{\prime}$. Dasselbe. Durch die verwendete Novokaindosis ist also der Zustand des desensibilisierten rechten Triceps nicht verändert.

7. " . Zustand unverändert.

8. " Dasselbe. Starre des linken Ellbogens $90^{\circ}$. Keine Starre im rechten Triceps. Das Tier kann auf dem rechten Vorderbein allein nicht stehen und knickt sofort ein, Sohlengang, Ataxie. Tonus des rechten Triceps sehr wechselnd, manchmal deutlich vorhanden, manchmal völlig fehlend.

8. $" 9 \mathrm{~h} 50^{\prime}$. In $\ddot{A}$ thernarkose dekapitiert. Äther abgestellt.

8. " $10^{\mathrm{h}} 10^{\prime}$. Sehr deutliche Starre im linken Triceps $\left(90^{\circ}\right)$. Keine Spur von Starre im rechten Ellbogen.

8. " $1 \mathrm{I}^{\mathrm{h}} 10^{\prime}$. Andauernd derselbe Zustand. Sehr deutliche Starre im linken Triceps, keine Spur von Starre im rechten Triceps.

8. " $11^{\mathrm{h}} 21^{\prime}$. Erstickung. Dabei prächtige steife Streckung des rechten Ellbogens durch Tricepskontraktion. Danach wieder künstliche Atmung. Starre links $90^{\circ}$, rechts Null.

Daraul werden, da nach Sherrington ${ }^{1}$ ) die proprioceptiven sensiblen Muskejnerven jeweils denselben Weg nehmen und in dasselbe Rückenmarkssegment eintreten wie die afferenten motorischen Nerven, die Vorderwurzeln nach Herausnahme des Rückenmarkes faradisch gereizt und der Effekt auf den blossgelegten Triceps beobachtet. Wirkungslos ist die Reizung von $\mathrm{C}_{4}, \mathrm{C}_{5}, \mathrm{C}_{6}, \mathrm{C}_{7}$ (vielleicht eine minimale Spur wirksam?) und $\mathrm{Th}_{2}$. Kontraktion des Triceps erfolgt bei Reizung von $\mathrm{C}_{8}$ (sehr stark) und von $\mathrm{Th}_{1}$ (deutlich).

Also ist durch die vorgenommene Hinterwurzeldurchschneidung: $\mathrm{C}_{7}-\mathrm{Th}_{2}$ der Triceps vollständig desensibilisiert gewesen.

1) C. S. Sherrington: Expts. in examination of the peripheral distribution of the fibres of the posterior roots of some spinal nerves. Trans. Roy. Soc. B. vol. 184 p. 641. London 1893. 
Ergebnis: Nach einseitiger Hinterwurzeldurehschneidung $\left(\mathrm{C}_{7}^{\prime}\right.$ bis $\mathrm{Th}_{2}$ ) tritt an dem zugehörigen Vorderbein Tonusverlust und Ataxie bei ungestörter aktiver Beweglichkeit ein. Das Tier kann auf dem Beine dauernd nicht allein stehen, wenn auch vorübergehend deutlicher Tonus im Ellbogen fühlbar ist. Nach beiderseitiger Einspritzung von Tetanustuxin in den Triceps heginnt auf der Normalseite nach 2 Tagen die lokale Starre, die in 6 Tagen allmählich an Stärke zunimmt. An der desensibilisierten Seite ist keine Tetanusstarre nachweisbar. Das Tier kann nicht einmal auf diesem Beine allein stehen, der Zustand des Triceps ändert sich während der Beobachtungsdauer nicht nachweislich. Einspritzung einer kleinen Novokaindosis ist auf der desensibilisierten Seite (5 Tage nach der Toxininjektion) ohne jede Wirkung auf den Triceps. Nach dem Dekapitieren zeigt der Triceps der Normalseite deutliche Tetanusstarre, während der Triceps auf der desenbilisierten Seite keine Spur von Starre zeigt, aber boi der Erstickung mit kräftiger Kontraktion reagiert.

Dieser Versuch zeigt auf das deutlichste, dass auch, wenn man den Schock der (einseitigen) Hinterwurzeldurchschneidung abklingen lässt und nun auf beiden Seiten gleiche Dosen von Tetanustoxin intramuskulär einspritzt, auf der Normalseite Starre eintritt, während sie innerhalb der Beobachtungszeit auf der desensibilisierten Seite ausbleibt.

Hieraus folgt, dass die tetanische Muskelstarre ganz oder wenigstens grösstenteils ausgelöst wird durch afferente Erregungen, die in das (durch das Tetanustoxin in den Zustand der t'bererregbarkeit versetzte) Zentralnervensystem einströmen.

Durch Hinterwurzeldurchschneidung werder sämtliche sensiblen Impulse, die von einem Körperabschnitte ausgehen, ausgeschaltet. Durch intramuskuläre Novokaineinspritzung werden nur die proprioceptiven Muskelnerven vorübergehend (und ohne dass die Möglichkeit von Schock besteht) gelähmt. Da auch hiernach (bei unveränderter zentraler und peripherer Motilität) die Starre ganz oder grösstenteils schwindet, so folgt, dass auch die Tetanusstarre, solange sie lokalisiert bleibt und nicht mit allgemeiner Reflexsteigerung gepaart geht, überwiegend unterhalten wird durch afferente proprioceptive Impulse, welche von den starren Muskeln selbst ausgehen. Wir haben also hier grundsätzlich denselben Vorgang wie beim Entstehen des normalen. Muskeltonus und der Enthirnungsstarre, nur dass der Zustand des Zentralorganes örtlich durch das Gift verändert ist ${ }^{1}$ ).

1) Im Zustande hochgradiger lokaler Tetanusstarre kann man durch passive Bewegungen des starren Gliedes häufig sehr deutliche „proprioceptive" Reflexe auf die anderen Gliedmaassen - Beuge- und Streckbewegungen auslösen. 
Dass der Angriffspunkt des Giftes bei der lokalen Muskelstarre wirklich das Zentrum und nicht etwa, wie Autokratow ${ }^{1}$ ) and Courmont und D o y $\circ \mathrm{n}^{2}$ ) wollten, die sensiblen Nervenenden im tetanuskranken Gliede sind, nehmen wir nach den Versuchen von Gumprecht ${ }^{3}$ ) und von Hans Meyer, Ransom und Fröhlich ${ }^{4}$ ) als feststehend an. Es folgt das daraus, dass Rückenmarksimpfung lokalen Tetanus erzengt, und zwar nach sehr viel kürzerer Inkubationszeit als bei peripherer Einspritzung, und dass nach Sperrung der Nerven mit Antitoxin periphere Tetanustoxininjektion keine lokale Starre mehr hervorruft.

Die oben geschilderten Versuche, nach denen Hinterwurzeldurchschneidung eine vorhandene Starre aufhebt und vorherige Hinterwurzeldurchschneidung den Eintritt der Starre verhindert, stehen im Einklang mit alten Versuchen von A utokratow ${ }^{1}$ ) und von Courmont und Doy on ${ }^{2}$ ). Dagegen liegen einige Versuche in der Literatur vor, die dem anscheinend widersprechen. Allerdings muss man hier alle diejenigen Experimente ausschalten, bei denen nach Hinterwurzeldurchtrennung das Toxin in das Rückenmark selbst eingespritzt wurde. Denn dann bekommt man das Bild des Tetanus dolorosus, das mit heftigen sensiblen Erregungen im Rückenmark selbst gepaart geht und daher für die Deutung des Entstehens des normalen lokalen Tetanus nicht herangezogen werden kann, bei dem der Tetanus dolorosus fehlt. Vielmehr kann man hier nur Versuche benutzen, in denen periphere Tetanusimpfung and Hinterwurzeidurchschneidung kombiniert wurden und danach lokaler Tetanus auftrat. Dieser Forderung genügen zwei Versuche:

1. Versuch von Gumprecht (Deutsche med, Woch. 1894, S. 546 und Pflüger's Archiv Bd. 59 S. 105. 1894). - Am 6. Februar werden einem Hunde von $6,5 \mathrm{~kg}$ auf der rechten Seite die Hinterwurzeln vom zweiten Lumbal- bis zum letzten Sacralsegment durchschnitten, was später bei-der Sektion kontrolliert werden konnte. Am 7. Februar wird in das asensible rechte Hinterbein das Filtrat einer Tetanusboullionkultur eingespritzt. D a dieses ohne deutlichen Effekt bleibt, erhält das Tier am 19. Februar eine nochmalige Injektion mit einer Tetanuskultur. Am 23. Februar findet sich das rechte Hinterbein in steifer Streckstellung, sinkt allerdings in der Ruhe herunter, geht aber sofort auf Händeklatschen und (am folgendén Tage) auf Beklopfen des linken sensiblen Hinterbeines in steifste Streckstellung über. Am 28. Februar allgemeiner Tetanus.

In diesem Falle blieb also die lokale Muskelstarre jm desensibilisierten Beine zunächst aus und traterstzusammen mit der allgemeinen Reflexsteigerung auf.

2. Versuch von Fröhlich und H. Meyer (Schmiedeberg's Arch. Bd. 79 S. 67. 1915). - Kleines Kätzchen. Am 6. Juni 1907 Durchschneidung aller Hinterwurzeln beiderseits vom zweiten Lumbal- bis zum letzten. Sacralsegment und des Filum terminale. - Am 10. Juni Ein-

1) Autokratow, Rech. expér. sur le mode de production des contractures dans le Tétanos. Arch. de méd. expér. t. 4 p. 700.1892.

2) Courmont u. Doyon, Méchanisme de production de contracture du Tétanos. Arch. de physiol. t. 25 p. 64.1893.

3) F. Gumprecht, Versuche über die physiol. Wirkungen des Tetanusgiftes im Organismus. Pflüger's Arch. Bd. 59 S. 105.1894.

4) H. Meyer u. F. Ransom, Unters. über den Tetanus. Schmiedeberg's Arch. Bd. 49 S. 369. 1903. - A. Fröhlich u. H. Meyer, Unters. über den Tetanus. Schmiedeberg's Arch. Bd. 79 S. 55. 1915. 
spritzung der enormen Dosis von $135000+$ ms Tetanustoxin in den linken Ischiadicusstamm. - Am 12. Juni das injizierte Bein stark extendiert, steif. Typischer lokaler Tetanus.

Leider fehlt in diesem Versuche die Kontrolle der Hinterwurzeldurchschneidung durch die Sektion oder eine genaue Sensibilitätsprüfung.

Diese Versuche zeigen, dass es gelingt, nach Hinterwurzeldurchschneidung durch maximale Tetanusvergiftung die Rückenmarkszentren in einen Zustand zu versetzen, dass sie auch von anderen afferenten Nerven aus erregt werden können, was natürlich hier nicht bestritten wird. Da aber die Kontrollimpfung mit Tetanusgift auf der anderen Seite in beiden Versuchen fehlt, so lässt sich nicht beurteilen, ob das Normalbein nicht früher oder stärker reagiert hätte. Die in unserer Arbeit bewiesene Tatsach $\theta$, dass durch Tetanustoxin vergiftete Rückenmarkszentren am schnellsten und leichtesten von den eigenen Proprioceptoren aus erregt werden, besonders wenn man mit mässigen Toxindosen arbeitet, wird durch diese Versuche nicht widerlegt.

Betrachtet man nun auf Grund der gewonnenen Resultate die Beobachtungen von E. Meyer und Weiler am Menschen, so ergibt sich, dass auch beim Menschen die Tetanusstarre, und zwar auch nach monate- und jahrelangem Bestehen, hauptsächlich durch proprioceptive Reflexe der starren Muskeln (Rectus abdominis, Biceps, Masseteren) selbst unterhalten wird; denn sie lässt sich durch intramuskuläre Injektion von Novokain bei unverändert erhaltener motorischer Innervation lösen.

Die Erklärungsversuche von E: Meyer und Weiler, dass Novokain eine unbekannte Wirkung auf den Muskel besitzt, und von H. Meyer und Fröhlich, dass durch die betreffenden Novokaininjektionen die gesamte motorische und sensible Innervation des Muskels ausgeschaltet sei, werden durch die hier erhaltenen Ergebnisse hinfällig und überflüssig.

Interessant ist, dass die Starre beim Menschen sich noch nach so langer Dauer (bis über 2 Jahre) durch kleine Novokaindosen unter Erhaltung der Motilität lösen lässt. Daraus kann man schliessen, dass es nicht zur sekundären peripheren Kontraktur gekommen ist, dass daher eine solche nicht aufzutreten braucht, ebenso wie auch wir bei unseren Experimenten niemals eine derartige Kontraktur gesehen haben.

Die in diesem Abschnitte geschilderten Versuche haben demnach zu folgendem Ergebnisse geführt:

Die lokale Tetanusstarre lässt sich sowohl am normalen wie am dekapitierten Tiere durch kleine Novokaindosen, welche die proprioceptiven Muskelnerven lähmen, die motorische Innervation aber quantitativ intakt lassen, ganz oder bis auf einen kleinen Rest aufheben. Dasselbe wird durch vor- oder nachherige Durchschneidung der 
Hinterwurzeln erreicht. Nach Durchtrennung der zugehörigen Hinterwurzeln ist intramuskuläre Einspritzung kleiner Novokaindosen in den tetanusstarren Muskel ohne jeden Einfluss. Die lokale Tetanusstarre wird zum grössten Teil bedingt bzw. unterhalten durch proprioceptive Impulse, welche von dem starren Muskel selbst dem durch das Tetanusgift affizierten Zentrum zufliessen. Die klinischen Beobachtungen von E. Meyer und Weiler über die Lösung der tetanischen Muskelstarre beim Menschen erklären sich ebenfalls durch die dabei eintretende Lähmung der sensiblen Muskelnerven.

\section{Weitere Beobachtungen über die tetanische Muskelstarre.}

Nachdem sich ergeben hatte, dass die tetanische Muskelstarre durch afferente, hauptsächlich proprioceptive Erregungen unterhalten wird, erhob sich erneut die Frage, wie sie sich in Allgemeinnarkose verhält. Schon Courmont und Doyon. ${ }^{1}$ ) sahen eine Abnahme des lokalen Tetanus in Chloroformnarkose, während andererseits Fröh lich und Hans Meyer ${ }^{2}$ ) in einer Äthernarkose, die so weit vertieft war, dass alle erkennbaren Reflexe schwanden, die lokale Tetanusstarre des Gastrocnemius der Katze erhalten fanden.

Nach unseren Erfahrungen verhält sich die Tetanusstarre bei der Äther- und Chloroformnarkose ungefähr so wie die "Narkosestarre“. Sie ist bei ausgesprochener Narkosestarre deutlich vorhanden, nimmt mit abnehmender Narkosestarre allmählich $a b$. und ist jedenfalls bis auf geringe Peste geschwunden, wenn die Narkosestarre bei Vertiefung der Narkose verschwindet. Es ist möglich, dass die Tetanusstarre in ihren letzten Resten noch etwas resistenter gegen die Narkose ist als die Narkosestarre, aber gross ist der Unterschied jedenfalls nicht.

Der Verhalten in der Chloroformnarkose erhellt aus folgendem. Versuchsbeispiel.

Katze von etwa $2 \mathrm{~kg}$.

3. März 1919. $1 \mathrm{ccm} 1 / 4 \%$ iges Tetanustoxin in den rechten Triceps.

5. ” Beginnende Starre.

6. " Deutliche Starre im rechten Triceps, der auf Pfotenkneifen noch vollständig gebeugt wird.

8. "Starke Starre im rechten Triceps, der nicht mehr vollständig ge" beugt wird. Leichte Starre der Schulterbeugei.

10. " Leichte Starre im Biceps.

13. " . Rechter Ellbogen beim Pfotenkneifen nur noch bis auf $90^{\circ}$ gebeugt. Passive Bewegungen der rechten Schulter bewirken proprioceptive

1) a. a. 0 .

2) A. Fröhlich u. H. Meyer, Über die Muskelstarre bei der Tetanusvergiftung. Münch. med. Woch. 1917, S. 289. 
Reflexe auf die Hinterbeine und das linke Vorderbein. Keine allgemeine Reflexsteigerung. Starre des rechten Triceps wird durch $2 \mathrm{ccm} \mathrm{1 \%}$ iges Novokain intramuskulär fast völlig gelöst.

15. März. Starke Starre des rechten Triceps, deutliche, aber geringere Starre des rechten Biceps, der. Schultermuskeln und der Beuger des Handgelenkes. Keine allgemeine Reflexsteigerung.

Nachmittag $3 \mathrm{~h} 10^{\prime}$. Tiefe Chloroformnarkose bis zum Erlöschen aller Reflexe. Tier völlig schlaff. Spontane Atmung. Starre des rechten Vorderbeines geschwunden. ( $\mathrm{CHCl}$-Gehalt des Blutes etwa $0,04-0,06 \%$.)

15. " 3 h 19'. Narkose beendet.

15. " 3 h $28^{\prime}$. Schwache Narkoselaufbewegungen. Linkes Vorderbein schlaff. Im rechten Triceps eine $\mathrm{Spur}$ Widerstand (ca. $0,036 \%$ ).

15. $" 3 \mathrm{~h} 30^{\prime}$. Beginn der Narkosestarre in den Hinterbeinen und im linken Trićeps. Schwache Starre im rechten Triceps (ca. 0,028\%).

Cornea- und Ohrreflex positiv, Patellarreflex schwach. Kurz darauf Beugereflex. Nunmehr ist die Starre im rechten Triceps deutlich (aber noch nichtstark), die anderen Gelenke des rechten Vorderbeines sind noch schlaff $(0,023 \%)$.

15. " $3^{\text {h }} 33^{\prime}$. Starre im linken Triceps deutlich, im rechten Triceps stark (nicht maximal). Schwache Starre in der rechten Schulter. Laufbewegungen (über $0,019 \%$ ).

15. " 3 br $35^{\prime}$. Starre im rechten Triceps ++ , in den rechten Schulterbeugern + , in den Schulterstreckern -, im rechten Biceps schwach.

Der linke Triceps hat deutliche, die linke Schulter schwache

Narkosestarre. Beugereflex und gekreuzter Streckreflex positiv.

In einem anderen Versuche wurde die Tetanusstarre des Triceps nach dreitägiger Dauer durch tiefe Chloroformnarkose bis zum Atemstillstand (ca. $0,06 \%$ Chloroform im Blute) vollständig aufgehoben, und war beim Wiedererwachen aus der Narkose im Stadium der Narkoselaufbewegungen bei vorhandener Narkosestarre des Quadriceps sehr stark ausgebildet $(0,02-0,028 \%$ Chloroform im Blute).

In diesen Versuchsprotokollen ist, auf Grund der Analysen von Storm van Leeuwen ${ }^{1}$ ) jedesmal der Chloroformgehalt des Blutes in Gewichtsprozenten angegeben, der bei den einzelnen Narkosegraden gefunden wird. Es ergibt sich, dass bei tiefer Narkose und schlaffer Muskulatur $(0,04-0,06 \%$ Chloroform) die Tetanusstarre verschwindet und etwa gleichzeitig mit der Narkosestarre oder, nur wenig früher (bei etwa $0,036-0,028 \%$ ) zurückkehrt. Bei etwa $0,02 \%$ Chloroform im Blute ist sie bei deutlicher Narkosestarre kräftig ausgesprochen.

In der Äthernaikose liegen die Verhältnisse etwas anders. Hier dauert nach den Feststellungen von Storm van Leeuwen ${ }^{2}$ ) die Narkosestarre viel länger und ist selbst beim Atemstillstand nicht immer völlig geschwunden. Der Atemstillstand tritt bei Katzen etwa

1) W. Storm van Leeuwen, Quantitative pharmak. Unters. uber die Reflexfunktionen des Rückenmarkes an Warmblütern. I. Mitt. Pflü ger's Arch. Bd. 154 S. 307. 1913. II. Mitt. Ibid. Bd. 159 S. 291. 1914. IV. Mitt. Bd. 165 S. 594.1916.

2) W. Storm van Leeuwen, III. Mitt. Pflüger's Arch. Bd. 165 S. 84. 1916. IV. Mitt. Ibid. Bd. 165 S. 594. 1916. 
bei $0,164 \%$ Äther im Blute ein, während die oberste Grenze für die Narkosestarre bei $0,18 \%$ liegt. Die Tetanusstarre verhält sich etwa ebenso. In einem Versuche war die Tetanusstarre noch vorhanden, als mit Äther bis zum Atemstillstand narkotisiert war. In einem anderen Falle wurde ebenfalls bis zum Atemstillstand narkotisiert, dabei schwand sowohl die Narkose- als auch die Tetanusstarre. In einem dritten Versuche atmete das Tier noch spontan, während sowohl die Narkose- als die Tetanusstarre gelöst waren; als dann in den Hinterbeinen wieder etwas Narkosestarre auftrat, kehrte auch die Tetanusstarre zurück, und als der Patellarreflex wieder auszulösen war, war die Tetanusstarre im Triceps sehr stark. Im allgemeinen lässt sich sagen, dass man durch Äther die Tetanusstarre nicht lösen kann, wenn man nicht ungefähr bis zum Atemstillstand oder über diesen hinaus narkotisiert.

Die Narkosestarre beruht bekanntlich auf der Lähmung der höheren Hirnteile und ist mit der Enthirnungsstarre Sherring ton's wesensgleich. Sie ist also wie diese hauptsächlich abhängig von den proprioceptiven Impulsen, die von den starren Muskeln selber ausgehen. Hierdurch wird es verständlich, dass die Tetanusstarre der Narkosestarre ziemlich genau parallel geht. Da nach der Tetanusvergiftung sich die Rückenmarkszentren in einem Zustande der Übererregbarkeit für proprioceptive Impulse befinden, so ist es nicht zu verwundern, dass bei der Narkose die Tetanusstarre, wenigstens in ihren letzten Resten, etwas später schwindet als die Narkosestarre der nichttetanischen Extremitäten.

Durch Fröhlich und H. Meyer ${ }^{1}$ ) ist gezeigt worden, dass der Warmblütermuskel im Zustande der Tetanusstarre bei elektrographischer Untersuchung stromlos ist. Semerau und Weiler ${ }^{2}$ ) haben kürzlich diesen Befund für den Menschen bestätigt. Als sich aus den hier geschilderten Versuchen ergeben hatte, dass die Tetanusstarre reflektorisch unterhalten wird, erschien es wünschenswert, das Phänomen aus eigener Anschauung kennenzulernen. Dank dem freundlichen Entgegenkommen von Professor J. K. A. Wertheim Salomonson in Amsterdam, der hierfür sein besonders empfindliches Instrumentarium zur Verfügung stellte und die Aufnahmen selbst machte, sind wir in der Lage, ebenfalls die Angabe von Fröhlich und Meyer zu bestätigen.

Versuch XXIV. Katze $3,6 \mathrm{~kg}$.

4. Jan. 1919. Einspritzung von je $1 / 2 \mathrm{ccm} 1 / 4 \%$ igem Tetanustoxin in der Triceps beider Vorderbeine.

7. "Beginnende Starre beiderseits.

1) A. Fröhlich und H. Meyer, Unters. über die Aktionsströme anhaltend verkürzter Muskeln. Zentralbl. f. Physiol. Bd. 26 S. 269.1912.

2) M. Semerau u. L. W eiler, Elektromyograph. Unters. am tetanischkranken starren Muskel d. Menschen. Zentralbl. f. Physiol. Bd. 33 S. 69. 1918. 
8. Jan. Deutliche Starre beiderseits. DieVorderbeine können auf Pfotenkneifen noch gebeugt werden.

Äthernarkose, die so weit vertieft wird, dass das Stadium der Narkoselaufbewegungen durchlaufen wird. Geringe Narkosestarre der Hinterbeine, bei Beugung im Knie tritt der erste fühlbare Widerstand bei $90^{\circ}$ auf. Der Quadriceps ist bei der elektrographischen Untersuchung völlig stromlos. Dabei liegt das Hinterbein in Streckstellung, der Quadriceps ist also nicht gespannt. Die Ableitung erfolgt mit unpolisierbaren Elektroden von der enthaarten befeuchteten Haut.

Bei dieser Tiefe der Narkose haben die beiden Triceps noch starke Starre. Ableitung mit unpolisierbaren Elektroden von der mit Strontiumsulfid enthaarten Haut über dem linken Triceps. Die eine Elektrode liegt uber dem distalen Muskelende, die andere auf zwei Drittel des Muskelbauches.

Empfindlichkeit der Saite $10 \mathrm{~mm}$ per Millivolt. Widerstand der Saite $9000 \mathrm{Ohm}$. Feldstärke ca. 32000 Gauss. Saitendicke $1,3 \mu$. Saitenlänge $5,6 \mathrm{~cm}$. Vergrösserung 1340 fach. Zeit ${ }^{1 / 100 ~ S e-~}$ kunde.

Auf der Aufnahme (Abb. 1) sieht man das Elektrokardiogramm. In den $Z$ wischenpausen ist die Saite völlig ruhig.

Eine zweite Aufnahme hatte genau das gleiche Ergebnis.

Wir kommen also zu dem überraschenden Ergebnis, dass im Zustande der Tetanusstarre der Muskel reflektorisch, und zwar hauptsä.chlich durch proprioceptive Impulse, in einen Zustand von Dauerverkürzung gerät, während welchem keine elektrischen Stromschwankungen vom Muskel abgeleitet werden können. Das Instrumentarium

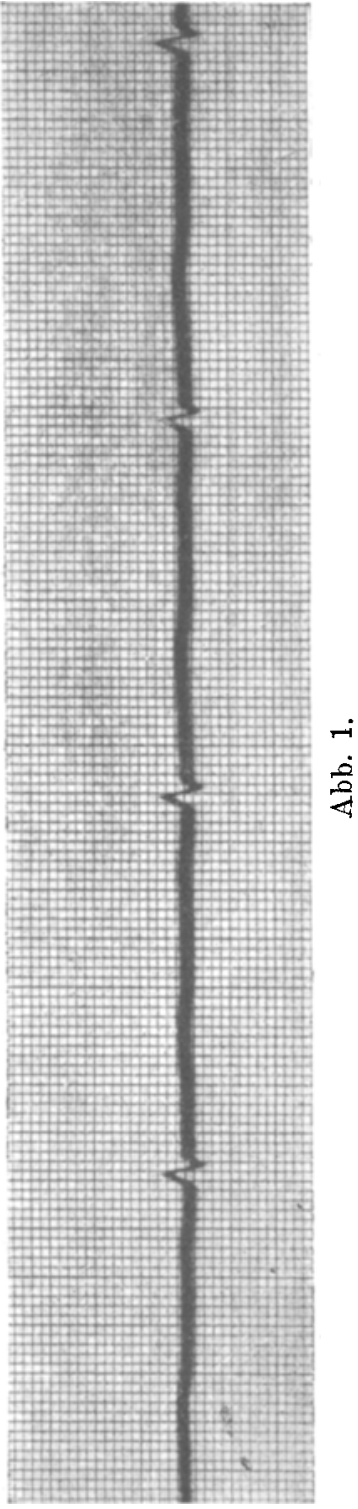

$\frac{\dot{0}}{\dot{0}}$ $\frac{\dot{0}}{4}$ war hinreichend empfindlich, um auch die kleinsten Schwankungen zu zeigen, wenn sie vorhanden gewesen wären; ist doch sogar das Elektrokardiogramm bei einfacher Ableitung vom Triceps deutlich zum Vorschein gekommen.

Dass ähnliche Verhältnisse auch bei reflektorischer Erregung menschlicher Muskeln ohne Tetanusvergiftung zu finden sind, lehrt eine kürzlich 
erschienene Mitteilung von Wertheim Salomonson ${ }^{1}$ ), welcher bei Patienten den Babinski'schen Reflex auslöste und gleichzeitig das Mechanogramm und Elektrogramm des Extensor hallucis longus aufzeichnete. Die Saite zeigt zunächst $0,11-0,66$ Sekunden lang lebhafte Schwingungen, und 0,10-0,14 Sekunden nach dem Beginn dieser Schwingungen fängt die Muskelkontraktion an, zuerst sehr langsam, dann sich steiler erhebend und schliesslich in einen 2-.. 3 Sekunden anhaltenden kräftigen Kontraktionszustand übergehend. Während dieses letzteren ist der Muskel völlig stromlos ${ }^{2}$ ).

Alle diese Tatsachen werden bei der so dringend nötigen Neubearbeitung der Physiologie des zentral innervierten Warmblütermuskels Berücksichtigung finden müssen. Vorläufig erscheint es noch verfrüht, sich in Hypothesen über die Deutung der Befunde zu ergehen.

Nur eine Tatsache mag in diesem Zusammenhange noch (anhangsweise) mitgeteilt werden. Bei einer Diskussion unserer Befunde äusserte ein Kollege, anknüpfend an die Lehre de Boer's von der Abhängigkeit des Muskeltonus vom Sympathicus, die Vermutung, dass die Tetanusstarre auf dem Wege über den Sympatbicus auf den Mưskel übermittelt werde. Auch E. Meyer und Weiler haben etwas Derartiges für möglich gehalten. Wir haben daher bei zwei Katzen mit voll entwickelter lokaler Tetanusstarre des Triceps, welche auch nach dem Dekapitieren kräftig erhalten blieb, das Ganglion stellatum der vergifteten Seite vom Rücken aus nach der Methode von Anderson ${ }^{3}$ ) exstirpiert. Danach blieb in beiden Fällen die Starre genau so stark wie vorher. Dagegen wurde der Triceps nach Durchschneidung des Brachialplexus völlig schlaff. Für eine sympathische Entstehung der Tetanusstarre hat.sich demnach - entsprechend unserer Erwartung - kein Anhaltspunkt gefunden.

\section{Zusammenfassung der Ergebnisse.}

Bei dezerebrierten Kalzen wird die Enthirnungsstarre des Triceps durch intramuskuläre Einsprilzling von kleinen Novokaindosen $(1 / 2-1 \mathrm{ccm}$ $1 \%$ bei Tieren von $1 \mathrm{~kg}$ ) hochgradig vermindert, aber in den meisten Fällen nicht völlig aujgehoben. Dabei ist die aktive Bewreglichkeit unverändert erhalten, und bei indirekter faradischer Reizung vom Plexus aus findet man dieselben Schwellenwerte wie am unvergifteten Muskel. Auch die tonischen Hals- und Labyrinthreflexe wirken noch auf den Triceps.

Erst beträchtlich grössere Novokaindosen $(4-8 \mathrm{~cm} 1 \%$ ) heben die

1) J. K. A. Wertheim Salomonson, Sur le réflexe de Babinski. Psychiatr. en Neurolog. Bladen 1918 (W inkler-Festschrift).

2) Anm. bei der Korrektur: Bornstein und Sänger (D. Z. f. Nervenheilkunde Bd. 52 S. 1. 1914) fanden in einem Falle von amyotrophischer Lateralsklerose den spastisch kontrakturierten Biceps ebenfalls stromlos.

3) H. K. Anderson, Journ. of Physiol. vol.31. 1904. Physiol. Soc. 21. Mai. 
indirekte Erregbarkeit des Muskels und damit die letzten Reste der Enthirnungsstarre und die aktive Beweglichkeit auf. Die direkte faradische Erregbarkeit des Muskels ist dann noch erhalten.

Durchschneidet man die Hinterwurzeln zu einem Vorderbein und erzeugt danach durch Dezerebrieren eine Enthirnungsstarre, an der sich das desensibilisierte Bein beteiligt, so ist intramuskuläre Einspritzung kleiner Novokaindosen ohne jede Wirkung auf die Starre. Erst grosse Novokaindosen, welche die indirekle Erregbarkeit vom Nerven aus beeinträchtigen, vermindern die Starre und heben sie auf.

Demnach lähmt Novokain, in kleinen Dosen intramuskulär eingespritzt, die proprioceptiven sensiblen Muskelnerven und vermindert dadurch die (reflektorisch bedingte) Enthirnungsstarre. Bis zu welchem Grade dieselbe abnimmt, hängt davon ab, in welchem Ausmaasse sich im Einzelfalle noch andere reflektorische Einflüsse am Zustandekommen der Starre des betreffenden Triceps beteiligen.

Novokain in grossen Dosen lähmt die motorischen Nervenenden im Muskel und hebt dodurch die Starre sowie die reflektorische und indirekte Erregbarkeit völlig auf. Die direkite Muskelerregbarkeit bleibt erhalten.

Einspritzung von kleinen Novokaindosen in den Triceps normaler Kalzen führt zu vorübergehendem Tonusverlusl bis zu völliger Erschlaffung bei gut erhallener aktiver Beweglichkeit. Es ergibt sich daraus eine sehr charakteristische Störung im Stehen und Laufen. Ataxie konnte nicht beobachtet werden.

Der normale Tonus des Triceps (und anderer Muskeln) wird hauptsächlich unterhalten von den proprioceptiven Erregungen, welche von diesen Muskeln selber ausgehen. Nach Hinterwurzeldurchschneidung bildet sich nach einiger Zeit ein Zustand aus, in welchem die motorischen Zentren auf sensible Impulse von anderen Körpergegenden erregbarer werden, so dass dann wieder ein gewisser Grad von reflektorischem Tonus möglich wird; dieser wird dann durch intramuskuläre Einsprilzung von kleinen Novokaindosen nicht weiter beeinträchtigt.

Nach intramuskulärer Einsprilzung von Tetanustoxin in den Triceps entwickell sich die lokale Starre anfänglich allein in dem injizierten Muskel. Dieser kann bei Beugebewegungen im Ellbogen zunächst noch aktiv erschlaffen, später ist das nicht mehr möglich. Danach werden auch der Biceps und die Schultermuskeln von Starre ergriffen. In allen beobachteten Fällen war die Starre rein zentral bedingt und nicht durch sekundäre periphere Kontraktur kompliziert.

Kleine Novokaindosen, welche die proprioceptiven Muskelnerven lähmen, die motorische Innervation aber völlig intakt lassen, heben die tetanische Muskelstarre im Triceps der Katze sowohl bei intakten wie bei dekapitierten Tieren total oder fast vollständig auf, wobei die aktive Beweglichkeit unvermindert erhalten bleibt. 
208 G. Liljestrand u. R. Magnus: Über die Wirkung des Novokains usw.

Auch durch Hinterwurzeldurchschneidung wird die ausgebildete lokale Tetanusstarre aufgehoben, während die Zentren des betreffenden Triceps reflektorisch und durch zentrale Erregungsmittel erregbar bleiben.

Wird nach einseitiger Hinterwurzeldurchschneidung gewartet, bis sich im desensibilisierten Bein wieder Tonus zu entwickeln beginnt, und spritzt man dann Tetanustoxin in den Triceps beider Vorderbeine, so entsteht nur in dem Arm mit erhaltener Sensibilität eine deutliche lokale Muskelstarre, während der desensibilisierte Triceps (am intakten Tier und nach dem Dekapitieren) bei erhaltener aktiver Beweglichkeit keine Tetanusstarre zeigt. Einspritzung einer kleinen Novokaindosis in den desensibilisierten Triceps ist dann wirkungslos.

Die lokale Muskelstarre beim Tetanus wird ausgelöst und unterhalten durch sensible Erregungen, welche grösstenteils in den starren Muskeln selbst ihren Ursprung nehmen, und welche deshalb zu der abnorm starken Muskelstarre führen, weil das Tetanusgift die Zentren in einen Zustand von Úbererregbarkeit versetzt hat. Die Wirkung des Novokains in kleinen Dosen beruht darauf, dass die proprioceptiven Erregungen aus dem injizierten Muskel aufgehoben werden, und dass dadurch die wichtigste Quelle für die lokale Starre abgeschnitten wird.

Aus einigen in der Literatur vorliegenden Versuchen lässt sich schliessen, dass man durch maximalste Tetanusvergiftung die Rückenmarkszentren in einen Zustand versetzen kann, in welchem sie auch von anderen afferenten Nerven aus in hochgradige Dauererregung versetzt werden können. Nach den von uns angewendeten Dosen trat dieses aber nicht ein.

Die klinischen Beobachtungen von Erich Meyer und Weiler, nach denen man mit Novokain die tetanische Starre auch beim Menschen lösen kann, ohne die aktive Beweglichkeit zu beeinträchtigen, findet durch diese Versuche ihre Erklärung. Aus ihnen ergibt sich ferner, dass die Tetanusstarre beim Menschen auch noch nach monate- und jahrelangem Bestehen durch proprioceptive Erregungen unterhalten wird, ohne dass es zu sekundärer Kontraktur zu kommen braucht.

Die Tetanusstarre verhält sich bei Allgemeinnarkose ungefähr wie die (ebenfalls durch proprioceptive Reflexe bedingte) ,Narkosestarre". Sie schwindet in tiefer Chloroformnarkose bei noch guter Almung, während sie in Athernarkose erst durch solche Dosen aufgehoben wird, welche die Atmung lähmen bzw. gefährden.

Während der reflektorisch ausgelösten tetanischen Dauerverkürzung des Triceps lassen sich im Muskel mil dem Saitengalvanometer keine Stromschwankungen nachweisen (Bestätigung der Beobachtungen von Fröhlich und H. Meyer).

Nach Exstirpation des Ganglion stellatum bleibt die lokale Tetanusstarre des Triceps unverändert bestehen. 